\title{
Split-Feed Counterflow Reverse Osmosis for Brine Concentration
}

\author{
by \\ Andrew Thomas Bouma \\ Submitted to the Department of Mechanical Engineering \\ in partial fulfillment of the requirements for the degree of \\ Master of Science in Mechanical Engineering \\ at the \\ MASSACHUSETTS INSTITUTE OF TECHNOLOGY \\ June 2018 \\ (C) Massachusetts Institute of Technology 2018. All rights reserved.
}

Author

Department of Mechanical Engineering

May 25, 2018

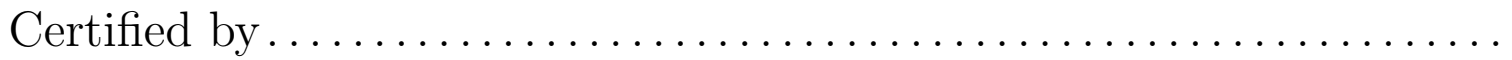

John H. Lienhard V

Abdul Latif Jameel Professor of Water

Thesis Supervisor

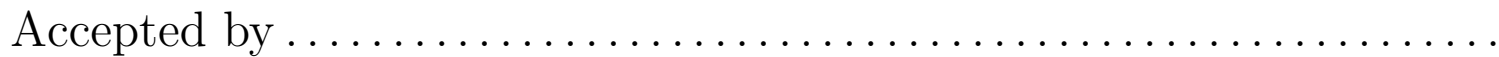

Rohan Abeyaratne

Chairman, Committee on Graduate Students 


\title{
Split-Feed Counterflow Reverse Osmosis for Brine Concentration
}

\author{
by \\ Andrew Thomas Bouma \\ Submitted to the Department of Mechanical Engineering
on May 25, 2018, in partial fulfillment of the
requirements for the degree of
Master of Science in Mechanical Engineering
}

\begin{abstract}
Brine concentration is a useful operation that allows for increased recovery ratios in water treatment systems, reduction of waste volumes, and the production of minerals from saline brines. As our world moves towards a more sustainable future, improvements in energy-efficient brine concentration will be important. While viable brine concentration technologies exist, current methods are often inefficient. In this thesis, a model is developed to simulate Counterflow Reverse Osmosis (CFRO), a membranebased, pressure-driven brine concentration technology. Using this model, a single CFRO module is simulated and its performance characterized. Entropy generation within a single-stage system is analyzed, which provides insights for configuring and optimizing multistaged systems. Additionally, a parametric analysis of membrane parameters provides direction for the development of CFRO-specific membranes. Two existing configurations of CFRO are discussed, and compared with a new third configuration, split feed CFRO, which is presented for the first time here. Split feed CFRO systems are simulated and optimized to provide guidance for system design. A variety of multistage systems operating at a range of recovery ratios are simulated, and the results compared are with existing desalination and brine concentration technologies. Potential is shown for the maximum recovery ratio of $\mathrm{RO}$ systems to increase significantly when hybridized with split-feed CFRO brine concentration systems, while the energy requirements of these hybridized systems is similar to, or an improvement on, the expected performance of conventional RO systems operating at high pressures and the same conditions. A large reduction in energy usage when compared to commonly used evaporative brine concentrators is shown to be possible.
\end{abstract}

Thesis Supervisor: John H. Lienhard V

Title: Abdul Latif Jameel Professor of Water 


\section{Acknowledgments}

May all glory, honor, and praise be to my faithful Lord and Savior Jesus Christ for His love and grace in the accomplishment of this thesis.

There are many individuals who deserve my deepest gratitude for their help and encouragement during the past two years, and I will thank just a few of them here.

I'd like to thank my advisor, Dr. John Lienhard V, for his guidance and insight that have added so much to my research, for giving me the opportunity to research and study under his direction, and for working so hard to put all of his students in a position to succeed at whatever their goals may be.

I'm grateful for all of those in the Lienhard Research Group, past and present, who have contributed to making the lab such an enjoyable and encouraging place to work and study. I have benefitted immensely by learning from and collaborating with so many knowledgeable and insightful people who are willing to take the time to share their gifts with me.

To those who have served as mentors to me, whether that be academically, professionally, or personally: Dr. Matthew Heun, Dr. Ren Tubergen, Jeff Davidson, Adam Rhodes, Ulrich Kuester, Brad Busscher, Dan Minikey, and many more, I thank you all from the bottom of my heart for giving your time and energy to teach me, for sharing your wisdom with me, and for challenging me to be the best I can be.

I would also like to thank my family and friends, who have loved and supported me in the good times and the bad, provided encouragement when I was struggling, and helped me to remember what is truly important in life. I thank Hope Fellowship Church and the early 20s Cafe group at Park Street Church for continually sharing the love and grace of Christ with me. I thank my friends, old and new, for the fun times we've shared together and for keeping me sane. I thank my siblings, Lindsey, Joel, Jessie, and Jake for their love, support, and for always knowing how to put a smile on my face. Finally, a most special thanks goes to my parents, Tom and Mary Bouma, who have been my biggest supporters for my entire life. I could not ask for better parents or role models. I aspire to be more like both of you, and I thank God 
for your presence in my life every day.

This research was funded by the Kuwait Foundation for the Advancement of Sciences (KFAS) through Project No. P31475EC01. I'm thankful for the support. 


\section{Contents}

1 Introduction $\quad 19$

1.1 Motivation . . . . . . . . . . . . . . . . . . . 19

1.2 Core technology . . . . . . . . . . . . . . . . . . . . . 21

1.3 Measures of system performance . . . . . . . . . . . . . . . 22

1.3.1 Recovery ratio. . . . . . . . . . . . . . . . . . . 22

1.3.2 Specific energy consumption . . . . . . . . . . . . . . 23

1.3.3 Second law efficiency . . . . . . . . . . . . . . . . . . 24

2 Counterflow Reverse Osmosis Model $\quad 27$

2.1 Model description . . . . . . . . . . . . . . . . . . . 27

2.2 Governing equations $\ldots \ldots \ldots \ldots \ldots \ldots$

2.3 Concentration polarization . . . . . . . . . . . . . . . . . . . 29

2.4 Membranes . . . . . . . . . . . . . . . . . . . . . 32

2.5 Pumping and work $\ldots \ldots \ldots \ldots \ldots \ldots \ldots \ldots \ldots \ldots$

2.6 Reverse osmosis model . . . . . . . . . . . . . . . . . . . . . 33

2.7 Model solution . . . . . . . . . . . . . . . . . . . . . . . 34

2.8 Validation . . . . . . . . . . . . . . . . . . . . . . . 34

2.9 Optimization schemes . . . . . . . . . . . . . . . . . . . 36

3 Single Module Analysis $\quad 37$

3.1 Base case . . . . . . . . . . . . . . . . . . . . . 37

3.2 Parametric analysis . . . . . . . . . . . . . . . . . . 39

3.2 .1 System parameters . . . . . . . . . . . . . . . . 39 
3.2.2 Membrane parameters .............. 45

4 CFRO Configurations $\quad 51$

4.1 Consecutive loop CFRO . . . . . . . . . . . . . . . . . . 51

4.2 Feed-through $\mathrm{CFRO} \ldots \ldots \ldots \ldots$. . . . . . . . . . . . 53

4.3 Split feed CFRO . . . . . . . . . . . . . . . . 55

5 Split Feed CFRO Simulations $\quad 59$

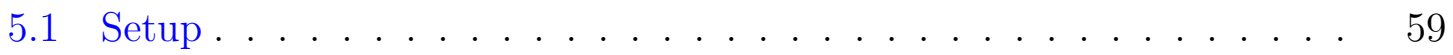

6 Results and Discussion $\quad 65$

6.1 Multistaged system results . . . . . . . . . . . . . . . 65

6.2 Varying B in a 6 -stage system . . . . . . . . . . . . . 69

$\begin{array}{lll}7 & \text { Summary and Conclusions } & 71\end{array}$ 


\section{List of Figures}

1-1 Diagrams of reverse osmosis, counterflow reverse osmosis, and forward osmosis modules along with the equations governing water flux through their membranes. The color intensity of the blue arrows correlates with stream solution concentration, while the green arrows show the direction of permeate flow. . . . . . . . . . . . . . .

2-1 Diagram of counterflow reverse osmosis model, broken into four elements. Mass flow rates of salt and water into and out of a single element are shown, on both sides of the membrane, along with water and salt flux through the membrane layer itself. . . . . . . . . . .

2-2 Diagram of concentration polarization within a membrane. Concentrations at relevant locations are depicted on the right, salt diffusion phenomena and directions governing concentrate-side ECP are shown on the left, and a representative concentration profile near and in the membrane is shown in the center. . . . . . . . . . . . . .

2-3 Comparison of data from CFRO models by Bartholomew et al. and the model presented in this paper. The concentration of the first sweep stream is plotted on the $\mathrm{x}$-axis, which is the concentration on the at the diluate side entrance of the first CFRO stage in the system. . . .

2-4 Comparison of data from RO experiments by Song et al. and the model presented in this paper. . . . . . . . . . . . . . . . 
3-1 Diagram of a single split-feed CFRO stage, showing the high pressure and booster pumps to the left of the membrane, and the circulation pump to the right, as well as the energy recovery device (ERD). The color intensity of the blue arrows correlates with stream solution concentration, while the green arrows show the direction of permeate flow. 38

3-2 Osmotic pressures shown along the length of the membrane in a single CFRO stage during base-case operation. . . . . . . . . . . . . . . 39

3-3 Osmotic pressures taken at one location in the module, the concentrate inlet, calculated at various membrane sizes, resulting in varying membrane flux. Inlet conditions and recovery ratio remain fixed in this case. The hydraulic pressure and $2^{\text {nd }}$ law efficiency are also shown. . .

3-4 Outlet concentrations and $2^{\text {nd }}$ law efficiency shown over a range of

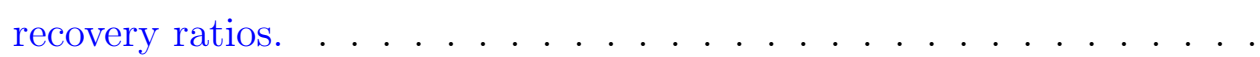

3-5 Stacked line graph showing the work into a CFRO system as a function of recovery ratio, broken down into the useful work performed and exergy destruction within different components in the system. . . . .

3-6 Stacked line graph showing the work into a CFRO system as a function of recovery ratio, shown at very low recovery ratios, broken down into the useful work performed and exergy destruction within different components in the system. . . . . . . . . . . . . . . . .

3-7 As the salt diffusion coefficient $B$ increases, the CFRO system's efficiency decreases, but the flux in the system increases due to the reduced internal concentration polarization. . . . . . . . . . . . . 46

3-8 The specific energy consumption (SEC) is reduced as the pure water permeability $A$ increases or the membrane structural parameter $S$ decreases. Asymptotic behavior is experienced when other mass transfer resistances in the system begin to dominate. . . . . . . . . . . . 
4-1 Diagram of a simple consecutive loop configuration with two CFRO stages and an RO stage. The dashed stream lines show the path of a single loop. The color intensity of the blue arrows correlates with stream solution concentration, while the green arrows show the direction of permeate flow. . . . . . . . . . . . . . . .

4-2 Diagram of a simple feed-through CFRO configuration with two CFRO stages and one RO stage. The color intensity of the blue arrows correlates with stream solution concentration, while the green arrows show the direction of permeate flow. . . . . . . . . . . . . . .

4-3 An unbalanced flux within CFRO stages is experienced in feed-through CFRO configurations due to unbalanced osmotic pressure changes on the two sides of the membrane, which arises because of imbalanced flow rates in the system. . . . . . . . . . . . . . . .

4-4 Diagram of a simple split feed CFRO configuration with two CFRO stages and a single RO stage. The color intensity of the blue arrows correlates with stream solution concentration, while the green arrows show the direction of permeate flow. . . . . . . . . . . 56

4-5 Diagram of a split feed CFRO configuration with a feed stream entering at a midpoint in the system. . . . . . . . . . . . . 57

5-1 Osmotic pressures within an optimized 3-stage split-feed system, plotted over the nondimensionalized length of the system. . . . . . . .

5-2 Diagram of a 3-stage split-feed CFRO system. The red dashed streams show a path of liquid recirculation. Increasing the flow rate in this loop can help to balance out the fluxes in the CFRO stages, but also increases pump work. . . . . . . . . . . . . . . .

5-3 Diagram of a 6 -stage split-feed CFRO system. . . . . . . . . . . . 
6-1 Specific energy consumption (SEC) for various recovery ratios and system sizes. High Pressure RO, labeled HPRO, shows to projected performance for conventional RO systems operating at pressures of greater than 70 bar. . . . . . . . . . . . . . . . . 66

6-2 Performance of selected systems, selected to show the most energy efficient configuration for each recovery ratio. High Pressure RO, labeled HPRO, shows projected performance for conventional RO systems operating at pressures of greater than 70 bar. . . . . . . . . . . .

6-3 Change in specific energy consumption (SEC) and applied hydraulc pressure in the first CFRO stage as the membrane's salt permeability

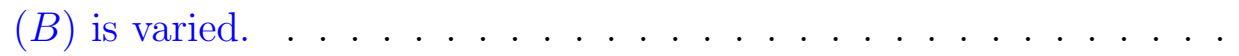




\section{List of Tables}

5.1 System parameters within the optimized 3-stage split feed CFRO system. 60

6.1 Operating parameters for a 6-stage split-feed system concentrating up to $200 \mathrm{~g} / \mathrm{kg} \ldots \ldots \ldots \ldots$. . . . . . . . . . . . . . . . . 68

6.2 Comparison of a 6-stage split feed CFRO system and 6-effect MVC system operating at the same conditions, both concentrating solutions

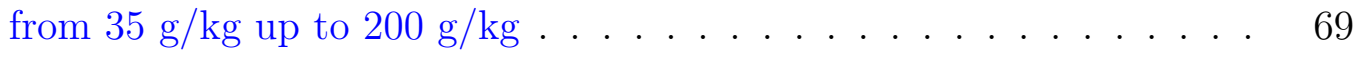


THIS PAGE INTENTIONALLY LEFT BLANK 


\title{
Nomenclature
}

\author{
Acronyms \\ CFRO counterflow reverse osmosis \\ COMRO cascading osmotically mediated reverse osmosis \\ ECP external concentration polarization \\ ED electrodialysis \\ EES Engineering Equations Solver \\ FO forward osmosis \\ HPRO high pressure reverse osmosis \\ ICP internal concentration polarization \\ MD membrane distillation \\ MVC mechanical vapor compression \\ OARO osmotically assisted reverse osmosis \\ RO reverse osmosis \\ RR recovery ratio \\ SEC specific energy consumption \\ ZLD zero liquid discharge
}

$\mathrm{kW} \cdot \mathrm{h} / \mathrm{m}^{3}$ 


\section{Greek Symbols}

$\begin{array}{llr}\Delta & \text { difference or change } & \mathrm{m} \\ \delta & \text { support layer thickness } & \text { W } \\ \dot{\Xi}_{d e} & \text { exergy destruction rate } & \mathrm{m} \\ \eta_{I I} & \text { isentropic (2 }{ }^{\text {nd }} \text { law) efficiency } & \mathrm{kg} / \mathrm{m}^{3} \\ \gamma & \text { concentrate side boundary layer thickness } & \\ \rho & \text { density } & - \\ \sum & \text { sum } & \\ \tau & \text { membrane tortuosity } & \text { - } \\ \varepsilon & \text { support layer porosity } & \end{array}$

\section{Roman Symbols}

$\begin{array}{llr}\dot{m} & \text { mass flow rate } & \mathrm{kg} / \mathrm{s} \\ \dot{V} & \text { volumetric flow rate } & \mathrm{m}^{3} / \mathrm{s} \\ A & \text { membrane permeability } & \mathrm{L} /\left(\mathrm{m}^{2} \cdot \mathrm{h} \cdot \mathrm{bar}\right) \\ A_{m e m} & \text { membrane area } & \mathrm{m}^{2} \\ B & \text { membrane salt permeability } & \mathrm{L} /\left(\mathrm{m}^{2} \cdot \mathrm{h}\right) \\ C & \text { concentration } & \mathrm{mol}^{2} \mathrm{~L} \\ D & \text { diffusion coefficient } & \mathrm{m}^{2} / \mathrm{s} \\ D_{h} & \text { hydraulic diameter } & \mathrm{m} \\ d x & \text { width of element } & \mathrm{m}\end{array}$




\begin{tabular}{|c|c|c|}
\hline$d y$ & distance perpindicular to membrane & $\mathrm{m}$ \\
\hline$f$ & friction factor & - \\
\hline$g$ & specific Gibbs free energy & $\mathrm{kJ} / \mathrm{kg}$ \\
\hline$J_{w}$ & water flux & $\mathrm{L} /\left(\mathrm{m}^{2} \cdot \mathrm{h}\right)$ \\
\hline$P$ & hydraulic pressure & bar \\
\hline$R e$ & Reynolds number & - \\
\hline$S$ & structural parameter & $\mathrm{m}$ \\
\hline$S c$ & Schmidt number & - \\
\hline$S h$ & Sherwood number & - \\
\hline$V$ & velocity & $\mathrm{m} / \mathrm{s}$ \\
\hline$W$ & work rate & $\mathrm{W}$ \\
\hline$w$ & concentration & $\mathrm{g} / \mathrm{kg}$ \\
\hline Flux $_{C F R O}$ & average flux of CFRO membranes & $\mathrm{L} /\left(\mathrm{m}^{2} \cdot \mathrm{h}\right)$ \\
\hline
\end{tabular}

\section{Superscripts}

$\begin{array}{ll}N & \text { number of stages } \\ \text { min } & \text { at zero recovery ratio }\end{array}$

\section{Subscripts}

$\begin{array}{ll}\text { actual } & \text { sum total } \\ \text { atm } & \text { atmospheric } \\ \text { brine } & \text { brine feed stream } \\ \text { bulk } & \text { in the bulk fluid }\end{array}$


c

\begin{tabular}{|c|c|}
\hline comp & ERD compression efficiency \\
\hline $\mathrm{d}$ & diluate side \\
\hline $\exp$ & ERD expansion efficiency \\
\hline feed & seawater feed stream \\
\hline $\mathrm{ICP}$ & due to internal concentration polarization \\
\hline in & flowing into the system \\
\hline least & useful work \\
\hline $\max$ & maximum \\
\hline mem & at the membrane-fluid interface \\
\hline mixing & due to mixing of permeated salt and water with diluate stream \\
\hline out & flowing out of the system \\
\hline permeate & permeate flow \\
\hline rec & recovered \\
\hline
\end{tabular}




\section{Chapter 1}

\section{Introduction}

\subsection{Motivation}

Brine management is becoming an increasingly important step in a complete water processing operation. Increased regulation [1, 2], environmental concerns $[1,3,4,5]$, and cost saving opportunities are all factors that have led to the growing demand for brine concentration technologies that can help to make water recycling feasible, reduce waste volume [6, 7], and in some cases recover value from brine streams [8]. Increased competition for clean water from a growing global population with agricultural and industrial needs, and additional stress on water supplies from climate change and changing diets has made access to clean water less secure and more expensive than in the past $[9,10,11]$, trends which are not likely to change in the near future. All of these factors are leading to increased adoption of brine concentration technologies and increased recovery ratios for water treatment systems already in place.

Recovering water from low-concentration solutions (below $70 \mathrm{~g} / \mathrm{kg}$ ) is most commonly done using reverse osmosis (RO) because of its high efficiency, reliability, and technical maturity $[12,13]$. Although RO is the dominant desalination technology today, it suffers from several challenges when operating at higher concentrations: the system's maximum recovery ratio is limited by a combination of feed concentration and membrane limitations, losses are greater in high recovery operations due to imbalances in driving pressures, and the rate of fouling increases at high concentration. 
Operating at high pressures also requires more expensive pumps, pressure vessels, and pipes, increasing system capital costs. Some of these challenges are being addressed by new configurations of RO technology, such as multi-stage RO, closed circuit RO, and batch $\mathrm{RO}[14,15,16,17,18]$, but some of these challenges remain.

In extreme cases, where no liquid waste can be disposed of due to regulations or high disposal costs, zero liquid discharge (ZLD) or minimum liquid discharge processes are necessary. Because of the limitations of current RO systems, further treatment of high-concentration solutions has been performed mainly by evaporative methods, such as mechanical vapor compression (MVC) [2], which is inefficient and energy intensive. Several other technologies, including electrodialysis (ED), forward osmosis (FO), membrane distillation (MD), and hybrid MVC-RO have been proposed as alternatives to MVC for high salinity brine concentration applications. Each of these technologies has its own benefits and drawbacks, and it is unlikely that one technology will be the optimal choice for all use cases. While each of these technologies has been explored in depth in the literature [13, 19, 20,21], we consider another emerging brine concentration technology that could compete with these alternatives.

Counterflow reverse osmosis (CFRO), which has also been called osmotically assisted reverse osmosis (OARO) [22] and cascading osmotically mediated reverse osmosis (COMRO) [23], has been shown to have the potential to be significantly more energy efficient than other brine concentration technologies, and also has the benefits of an increased operating range compared to RO. This is because CFRO's recovery ratio can be increased without requiring higher pressures and fluxes, as additional stages operating at low hydraulic pressure differences can be employed to extract more water from a brine stream. Additionally, CFRO's ability to operate multiple stages at low hydraulic pressure differentials instead of a single stage at a much higher hydraulic pressure differential may reduce the propensity for fouling, which is another potential benefit of the technology [24]. 


\subsection{Core technology}

At its core, CFRO is a membrane-based, pressure driven brine concentration technology, which shares many properties with other membrane-based chemical separation technologies, such as RO and FO. Like RO, permeate flows from the side of high concentration and high hydraulic pressure, across the membrane, to the side of low concentration and low hydraulic pressure. However, unlike RO, CFRO employs two feed streams instead of one. The first feed stream, which is referred to as the concentrate stream, is analogous to the feed stream in an RO system. The concentrate stream is dewatered as permeate flows through the semi-permeable, salt-rejecting membrane, and leaves the module with a reduced mass flow rate and increased concentration. On the opposite side of the membrane is another saline feed stream, which we call the diluate stream. This stream is diluted as permeate flows through the membrane, and the stream leaves the module with increased mass flow rate and reduced concentration.

Flow through the membrane is governed by the familiar solution diffusion equation [25]:

$$
J_{w}=A\left[\left(P_{c}-P_{d}\right)-\left(\pi_{c}-\pi_{d}\right)\right]
$$

where $J_{w}$ is the water flux through the membrane, $A$ is the membrane's water permeability, $P$ is hydraulic pressure, $\pi$ is osmotic pressure, and subscripts $c$ and $d$ denote the concentrate and diluate sides of the membrane, respectively. Another important aspect of this technology is that the feed streams flow in opposite directions. This allows for the balancing of osmotic pressure differences across the membrane, which leads to increases in efficiency by reducing entropy generation, much in the same way that balancing temperature differences in a counterflow heat exchanger increases the efficiency of heat transfer [26, 27]. Figure 1-1 shows a comparison of single stages of $\mathrm{RO}, \mathrm{FO}$, and CFRO systems along with the equations that govern water flux through the membrane in each system.

CFRO modules can be configured in several different cascading configurations to produce product streams at a wide variety of conditions. CFRO can also be 


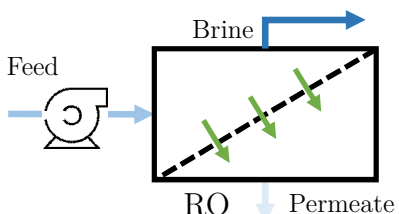

(a) Reverse osmosis $J_{w}=A(P-\pi)$

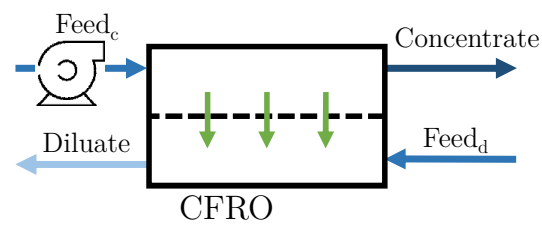

(b) Counterflow reverse osmosis $J_{w}=A(\Delta P-\Delta \pi)$

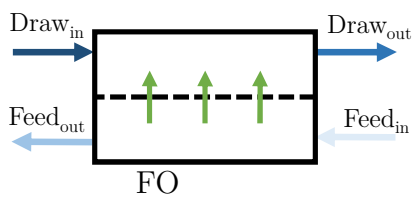

(c) Forward osmosis $J_{w}=A \Delta \pi$

Figure 1-1: Diagrams of reverse osmosis, counterflow reverse osmosis, and forward osmosis modules along with the equations governing water flux through their membranes. The color intensity of the blue arrows correlates with stream solution concentration, while the green arrows show the direction of permeate flow.

hybridized with a normal RO system to produce pure water in addition to highly concentrated brines. Some of these configurations are discussed in Chapter 4 of this thesis. However, before looking at more complex configurations, we first define several measures of system performance, and then seek to understand the operation of a single stage using a one-dimensional finite difference computational model. This model is explained in depth in Chapter 2.

\subsection{Measures of system performance}

There are three ways to quantify the performance of brine concentration systems that will be used in this work: Recovery ratio $(R R)$, specific energy consumption $(S E C)$, and 2nd law efficiency $\left(\eta_{I I}\right)$.

\subsubsection{Recovery ratio}

The recovery ratio, $R R$, can be defined in several different ways depending on the system being analyzed. In a system that has one feed stream, one brine stream, and one permeate stream, the recovery ratio is defined as:

$$
R R=\frac{\dot{m}_{\text {permeate }}}{\dot{m}_{\text {feed }}}
$$

where $\dot{m}_{\text {permeate }}$ is the mass flow rate of permeate through the membrane and $\dot{m}_{\text {feed }}$ is the mass flow rate of the single feed stream. However, when looking at a single 
CFRO exchanger, there are two feed streams and two outlet streams. Because the feed streams do not have to be at the same concentration, we define the recovery ratio as the amount of permeate recovered from the concentrate side feed stream:

$$
R R=\frac{\dot{m}_{\text {permeate }}}{\dot{m}_{\text {feed }, c}}
$$

where $\dot{m}_{f e e d, c}$ is the mass flow rate at the inlet of the concentrate side feed stream.

The recovery ratio quantifies the fraction of feedwater that is recovered or passes through the membrane. For situations where the goal is to achieve zero or minimum liquid discharge, achieving high recovery ratios is very important, as it reduces the work that needs to be done by secondary processes to concentrate brine to saturation levels. In situations where achieving high concentrations or minimizing waste volumes is of less concern, some measure of energy efficiency will most likely be more important.

\subsubsection{Specific energy consumption}

Specific energy consumption, $S E C$, is defined as the amount of energy used to create one unit of product. This is a simple way to approximate the efficiency of a system. For a system with one feed, one brine, and one permeate stream, the $S E C$ is defined as:

$$
S E C=\frac{W}{\dot{V}_{\text {permeate }}}
$$

where $W$ is the total electrical work input into the system, and $\dot{V}_{\text {permeate }}$ is the volumetric flow rate of the permeate stream, usually measured in $\mathrm{m}^{3}$. For a single CFRO module with two feed and two outlet streams, the definition of $S E C$ is the same, but

care should be taken to ensure that the $\dot{V}_{\text {permeate }}$ considered is the volumetric flow rate of permeate that passes through the membrane, and not the volumetric flow rate of one of the feed or outlet streams.

Specific energy consumption gives a simple way to compare the amount of energy used between systems, although it does not provide a complete picture. Because it is 
easier to recover pure water from low concentration streams than high concentration streams, it is not advised to compare systems that have different recovery ratios or feed conditions using $S E C$. This would constitute comparison of systems that perform different amounts of thermodynamically useful work.

\subsubsection{Second law efficiency}

$2^{\text {nd }}$ law efficiency, $\eta_{I I}$, is the ratio of thermodynamic least work for a process compared to the actual work taken into the system:

$$
\eta_{I I}=\frac{W_{\text {least }}}{W_{\text {actual }}}
$$

where the least work $W_{\text {least }}$ is the work done by a thermodynamically reversible process to perform the operation specified, which in this case is a chemical separation. The least work, also called the least work of separation or useful work done, is defined as

$$
W_{\text {least }}=\sum \dot{m}_{\text {out }} g_{\text {out }}-\sum \dot{m}_{\text {in }} g_{\text {in }}
$$

where $g$ is the Gibbs free energy of the inlet or outlet stream, and $\dot{m}$ is the stream's mass flow rate [12]. Within the realm of desalination, there is still some difference of opinion as to how to define least work. When the sole goal of a process is to produce pure water, and the brine is considered useless waste, the least work is often defined as $W_{\text {least }}^{\text {min }}$, the minimum work required to separate water from the feed stream at a recovery ratio approaching zero. This definition is commonly chosen because, if the sole purpose of the plant is to produce water, the customer generally does not care what the recovery ratio of the plant that produced it was. Any given drop of water is agnostic as to how it was produced. Therefore, it makes sense that the minimum energy to produce the water is considered regardless of the recovery ratio. Because the first drop taken from a saline feed stream will have the lowest energy requirement to be extracted, the minimum energy requirement will be when the recovery ratio approaches zero [12]. 
However, because we are interested in potentially recovering products from the brine streams in minimum or zero liquid discharge systems, the recovery ratio is important. For a brine concentrator, we care about both the energy to produce the products, and the state or concentration of both the concentrate and diluate streams. Therefore, for this analysis, we take the least work to be the least work at a finite recovery ratio [28]. This will allow us to compare the work done in the actual system to the work that would be required by a thermodynamically reversible system operating at the same inlet and outlet conditions. Further discussion of thermodynamic least work and $2^{\text {nd }}$ law efficiency can be found in references $[12,29,30]$. 
THIS PAGE INTENTIONALLY LEFT BLANK 


\section{Chapter 2}

\section{Counterflow Reverse Osmosis}

\section{Model}

In order to better understand the physical picture of what will occur in a CFRO system, a computational model is developed, beginning with a model of a single CFRO module, and scaling up to multistage systems.

\subsection{Model description}

We modeled each CFRO module as a spiral wound membrane with counter-current flow. The model uses a finite difference approach with 100 elements per stage, although element count was reduced for some multistage configurations because of computer program limitations. The model is solved iteratively using Engineering Equations Solver (EES). EES was chosen for this application because of its simplicity, its useful optimization techniques, and its built-in property tables [31, 32]. While seawater property packages are valuable for situations involving low concentrations, there is not enough data on seawater behavior at high concentrations to use these packages for all cases. Therefore, we chose to use the Pitzer model for $\mathrm{NaCl}$ solutions [33] to simulate $\mathrm{NaCl}$ solutions instead of seawater solutions. While seawater properties could have been used for some low concentration simulations, $\mathrm{NaCl}$ solutions are used for all simulations in this study for the sake of uniformity. 


\subsection{Governing equations}

Within each differential element, mass flow rates of salt and water are calculated using mass conservation equations. The mass flow rate of water into and out of each element is balanced with the water flux through the membrane. This balance is performed on both sides of the membrane. The same mass balance is performed for salt. A diagram of the setup is shown in Figure 2-1. Pressure drop along the

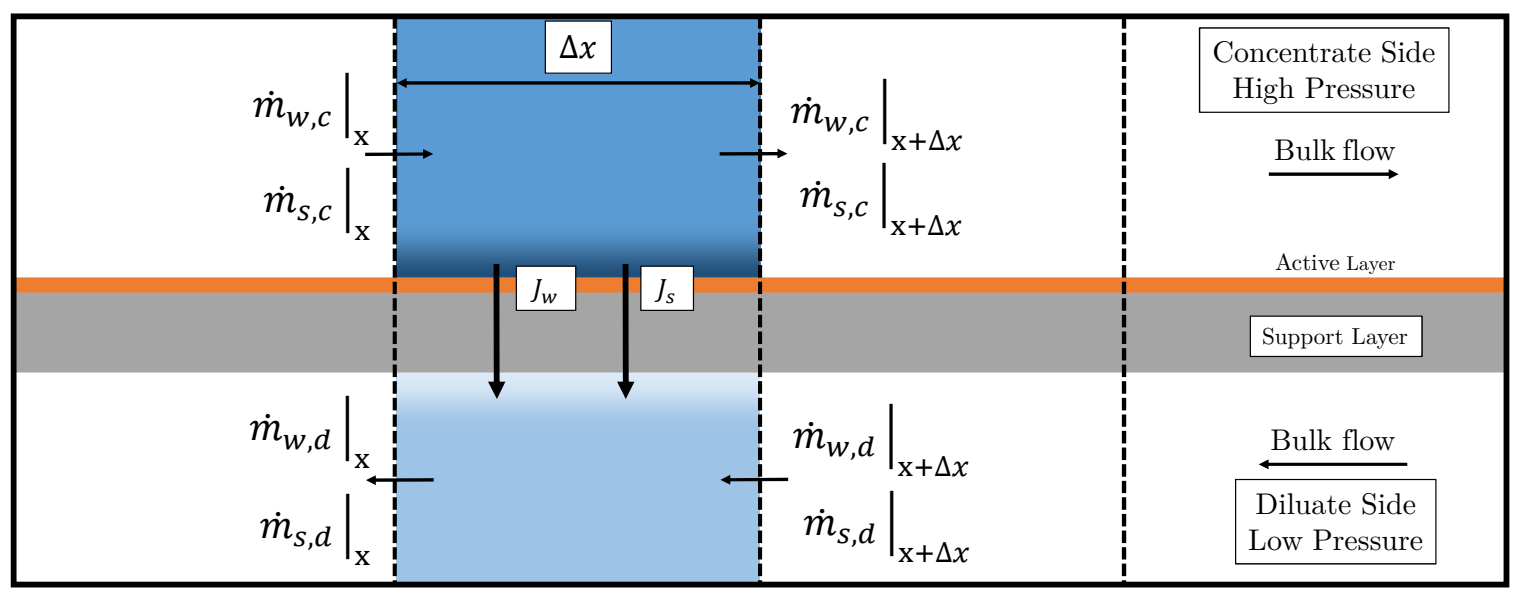

Figure 2-1: Diagram of counterflow reverse osmosis model, broken into four elements. Mass flow rates of salt and water into and out of a single element are shown, on both sides of the membrane, along with water and salt flux through the membrane layer itself.

module is estimated using the Darcy-Weisbach equation, along with a friction factor correlation specific to membrane systems [34].

$$
\begin{gathered}
\Delta P=f \frac{d x}{D_{h}} \rho \frac{V^{2}}{2} \\
f=3.23 R e^{-0.3}
\end{gathered}
$$

With the hydraulic pressure and mass flow rates of salt and water known, the osmotic pressure, viscosity, density, Gibbs free energy, and other relevant values can be calculated using the Pitzer model [33]. Water flux through the membrane is governed by the solution diffusion model [25], and salt rejection takes place at the 
active layer of the membrane, giving the equation:

$$
J_{w}=A\left[\left(P_{c}-P_{d}\right)-\left(\pi_{c, m e m}-\pi_{d, s u p}\right)\right]
$$

where $\pi_{c, m e m}$ is the osmotic pressure on the concentrate side, at the membrane surface,

and $\pi_{d, \text { sup }}$ is the osmotic pressure on the diluate side of membrane's active layer, at the interface between the support layer and active layer. Salt flow through the membrane is governed by the salt permeability coefficient, $B$, and the difference in concentration of salt across the active layer

$$
J_{\text {salt }}=B\left(C_{c, \text { mem }}-C_{d, \text { sup }}\right)
$$

where $J_{\text {salt }}$ is the salt flux, $C_{c_{m e m}}$ is the solution concentration on the concentrate side of the membrane, at the membrane surface, and $C_{d, \text { sup }}$ is the solution concentration on the diluate side of the membrane's active layer, at the interface between the support layer and the active layer of the membrane. The solution concentration is taken at these points because only the active layer of the membrane actually performs the separation of salt and water.

\subsection{Concentration polarization}

There are three relevant modes of mass transfer near the active layer of the membrane which describe the salt concentration in a thin concentration boundary layer. In a layer near the membrane surface on the concentrate side of the membrane, salt is rejected while water passes through the membrane. This creates a buildup of salt near the membrane surface, and the resulting concentration gradient leads to salt diffusion away from the membrane by molecular diffusion. At the membrane surface, this molecular diffusion of salt is balanced by the salt diffusing through the membrane, governed by Equation 2.4, and salt advection toward the surface, carried by the water flowing through the membrane.

On the diluate side of the membrane, the same phenomena govern salt mass 


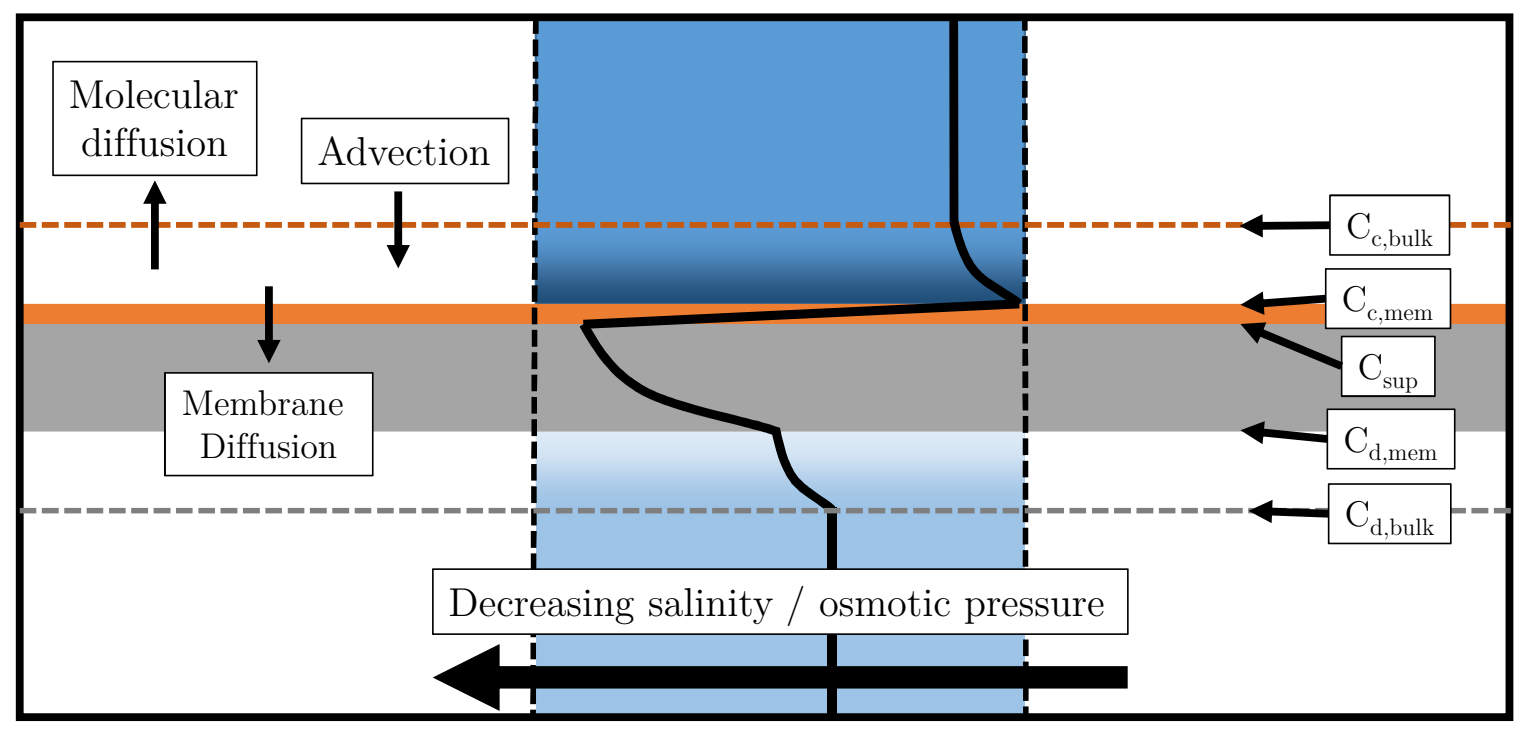

Figure 2-2: Diagram of concentration polarization within a membrane. Concentrations at relevant locations are depicted on the right, salt diffusion phenomena and directions governing concentrate-side ECP are shown on the left, and a representative concentration profile near and in the membrane is shown in the center.

transport again. The solution that flows through the active layer is more dilute than the bulk solution on the diluate side of the membrane, which causes salt to diffuse towards the membrane, while advection carries salt away. The changes in concentration due to these effects, which is called concentration polarization, occur in three different control volumes; one on the concentrate side of the membrane and one on the diluate side of the membrane just outside the support layer, which are called external concentration polarization (ECP) as they are external to the membrane, and another within the support layer itself, which is referred to as internal concentration polarization (ICP). These effects are shown in Figure 2-2 While the effect of ECP on the dilulate side of the membrane is often ignored due to being small compared to ICP, we include it here and for single module analyses. It has been shown that as membrane technology improves, accounting for the boundary layers outside the membrane becomes more important [35, 36]. However, for multistage cases where diluate-side ECP is negligible compared to other losses in the system, it is ignored in order to reduce computation time and complexity.

The mass balance equations for salt flow result in three coupled differential equa- 
tions with three unknown values: $C_{d, m e m}, C_{\text {sup }}$, and $C_{c, m e m}$, which are the concentrations on the diluate side of the membrane at the support layer surface, the concentration at the interface between the support layer and active layer, and the concentration at the membrane surface on the concentrate side, respectively.

$$
\begin{aligned}
& J_{w} C_{c, \text { bulk }}=B\left(C_{c, \text { mem }}-C_{\text {sup }}\right)+D \frac{d C}{d y} \\
& J_{w} C_{d, \text { mem }}=B\left(C_{c, \text { mem }}-C_{\text {sup }}\right)+D \frac{d C}{d y} \\
& J_{w} C_{d, \text { bulk }}=B\left(C_{c, \text { mem }}-C_{\text {sup }}\right)+D \frac{d C}{d y}
\end{aligned}
$$

These equations are solved using the known bulk concentrations on the concentrate and diluate sides as boundary conditions, resulting in three more coupled equations.

$$
\begin{aligned}
& C_{C, \text { bulk }}=\frac{B\left(C_{\text {mem }, c}-C_{\text {sup }}\right)}{J_{w}}\left[1-\exp \left(-\frac{J_{w} \gamma}{D}\right)\right]+C_{C, \text { mem }} \cdot \exp \left(-\frac{J_{w} \delta}{D}\right) \\
& C_{D, \text { mem }}=\frac{B\left(C_{C, \text { mem }}-C_{\text {sup }}\right)}{J_{w}}\left[1-\exp \left(-\frac{J_{w} \zeta}{D}\right)\right]+C_{D, \text { bulk }} \cdot \exp \left(-\frac{J_{w} \zeta}{D}\right) \\
& C_{\text {sup }}=\frac{B\left(C_{C, \text { mem }}-C_{\text {sup }}\right)}{J_{w}}\left[1-\exp \left(-\frac{J_{w} S}{D}\right)\right]+C_{D, m e m} \cdot \exp \left(-\frac{J_{w} S}{D}\right)
\end{aligned}
$$

The above equations can be solved to provide the concentration profile throughout the system, a depiction of which is shown in Figure 2-2. In the above equations, $D$ is the diffusion coefficient, $\gamma$ is the boundary layer thickness on the concentrate side, $\zeta$ is the boundary layer thickness on the diluate side, and $S$ is the structural parameter of the membrane support layer. $S$ is a function of the membrane's thickness $\delta$, porosity $\epsilon$, and tortuosity $\tau$ :

$$
S=\frac{\delta \tau}{\epsilon}
$$

The thickness of the concentration boundary layer on either side of the membrane is determined using a Sherwood number correlation [37].

$$
S h=0.2 R e^{0.57} S c^{0.4}
$$


It is worth noting that the degree of ICP is a strong function of the membrane structural parameter $S$ and the water flux through the membrane $J_{w}$. A significant increase in flux will lead to greatly reduced osmotic pressures at the interface between the active and support layers of the membrane, which will in turn lead to requirements for much higher trans-membrane pressure in order to achieve the desired flux.

\subsection{Membranes}

The membranes considered for this model are based off of the HTI-TFC membranes investigated by Straub et al. [38] with properties $A=2.49\left[\mathrm{~L} / \mathrm{m}^{2}\right.$-h-bar], $B=0.39$ $\left[\mathrm{L} / \mathrm{m}^{2}-\mathrm{h}\right], S=564 \times 10^{-6} \mathrm{~m}$, and a burst pressure of 50 bar.

As will be shown in Section 3.2.2, CFRO performance is sensitive to the membrane structural parameter, and so membranes with high permeability and low structural parameter are desirable. While no manufacturer makes membranes specifically for CFRO as of the time of this writing, the next best option may be membranes designed for FO or pressure retarded osmosis (PRO), which are designed to have a structural parameter much lower than RO membranes. However, FO membranes are not designed to withstand the high pressures that may be used in CFRO. Thus, the we have performed all computations in this work under the assumption that with some development, a membrane with the same $A, B$, and $S$ values as the FO membrane cited above could be built with a higher maximum operating pressure of 70 bar. The membranes are assumed to be in a spiral-wound configuration, with spacer properties from Schock and Miquel [34]. Successful operation may still be achieved with RO membranes, but their design may also lead to increased energy consumption, and complications at high concentrations and fluxes. 


\subsection{Pumping and work}

With all conditions inside the module found, work input can be calculated. Pump work is determined using the equation

$$
W=\frac{\dot{V} \Delta P}{\eta_{\text {pump }}}
$$

where all pumps are assumed to have an efficiency of $80 \%$. Depending on how a configuration is arranged, there may be up to three different pumps per stage, including a high pressure pump, a circulation pump to overcome the drag on the diluate side of the membrane, and a booster pump to bring up the pressure of the stream coming out of an energy recovery device (ERD) to the high pressure required at the concentrate inlet. ERD's can take various forms, such as isobaric energy recovery devices, Pelton wheels, or turbochargers, and they operate between the concentrate outlet and the concentrate feed streams. A diagram showing a possible setup for a CFRO system is shown in Figure 3-1. The equation governing the recovered energy from an isobaric energy recovery device is given by Mistry et al. [12] and shown below:

$$
P_{\text {rec }}=P_{\text {feed }}-\eta_{\text {exp }} \eta_{\text {comp }}\left(\frac{\rho_{\text {feed }}}{\rho_{\text {brine }}}\right)\left(P_{\text {brine }}-P_{\text {atm }}\right)
$$

Like Mistry, we assume both the expansion and compression processes in the ERD to be $98 \%$ efficient.

\subsection{Reverse osmosis model}

In many instances, configurations of cascading CFRO stages dilute the water to a point where it is treatable with reverse osmosis. In order to simulate RO using the model developed above, several modifications are made. First, the direction of flow is switched so that the feed and permeate streams flow in parallel. Next, the mass flow rate at the inlet of the diluate stream is set to zero. Finally, the membrane properties are changed to better reflect the properties of real RO systems. The membrane 
properties are based upon DOW Filmtec SWRO membranes with $99.4 \%$ rejection [39].

\subsection{Model solution}

For a single module, 7 parameters must be chosen to fix the system. Inputs include mass flow rates for both the concentrate and diluate inlet streams, as well as concentration for each inlet stream. Other variables that are required include the total membrane area and another dimension which fixes the width or length of the membrane module. This can be done either by picking the membrane dimension itself, or by specifying the flow velocity at some point in the module. For example, if the inlet mass flow rate and velocity are specified, because the channel height is already specified, the membrane's width is prescribed. Finally, one more value must be specified to determine the "duty", or amount of useful work done in the system. This can be an outlet concentration for either stream, a recovery ratio, or the applied hydraulic pressure. This final value fixes all other conditions within the module. With all required variables specified, the system is numerically solved using EES.

\subsection{Validation}

Validation for this model proved difficult because of the lack of published CFRO experimental data. We chose to validate against two different sources, one being data from RO experiments, the other being another published CFRO model. To validate against CFRO data, we set up the system as described by Bartholomew et al. [22], substituting their membrane properties and feed conditions. We also set the $B$ value of the membrane to zero, in order to match their assumption of no salt flux through the membrane. To compare our model to RO data, we used the RO model described above in Section 2.6. Membrane parameters were changed to match those of the RO membranes used in the study to which we compared. Results from the CFRO simulations were compared to experimental data published by Song et al. [40]. 


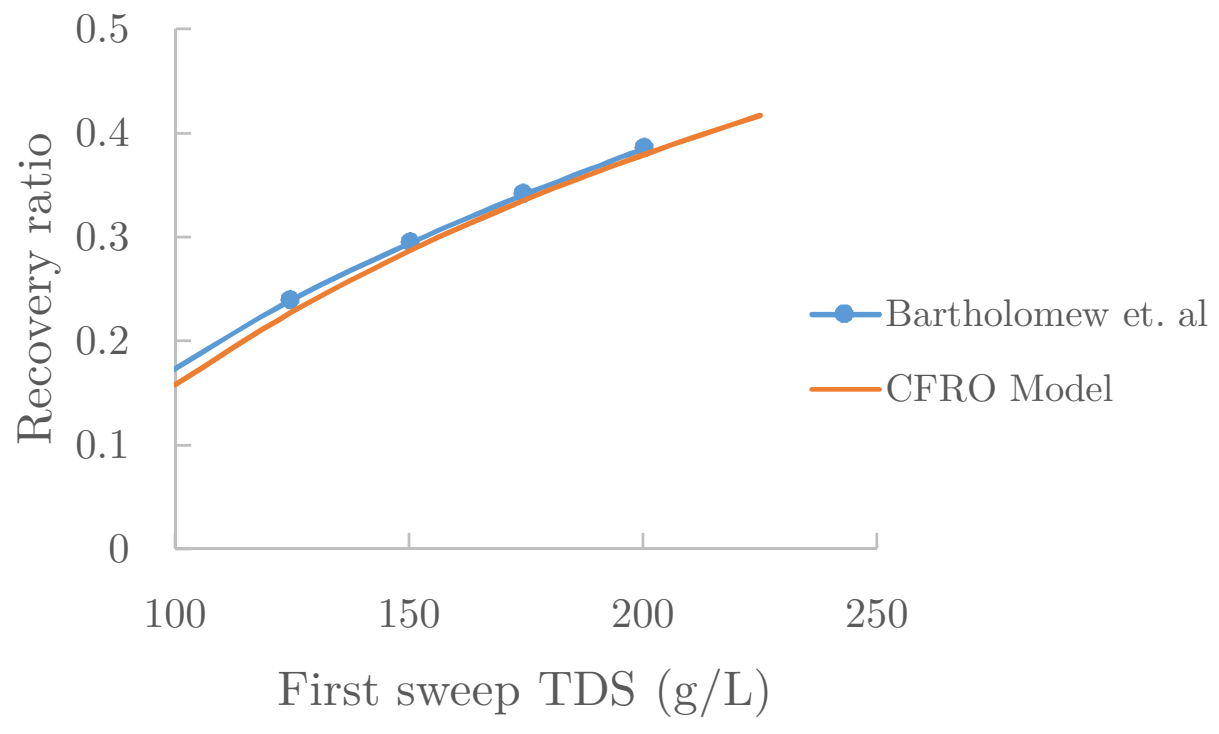

Figure 2-3: Comparison of data from CFRO models by Bartholomew et al. and the model presented in this paper. The concentration of the first sweep stream is plotted on the $\mathrm{x}$-axis, which is the concentration on the at the diluate side entrance of the first CFRO stage in the system.

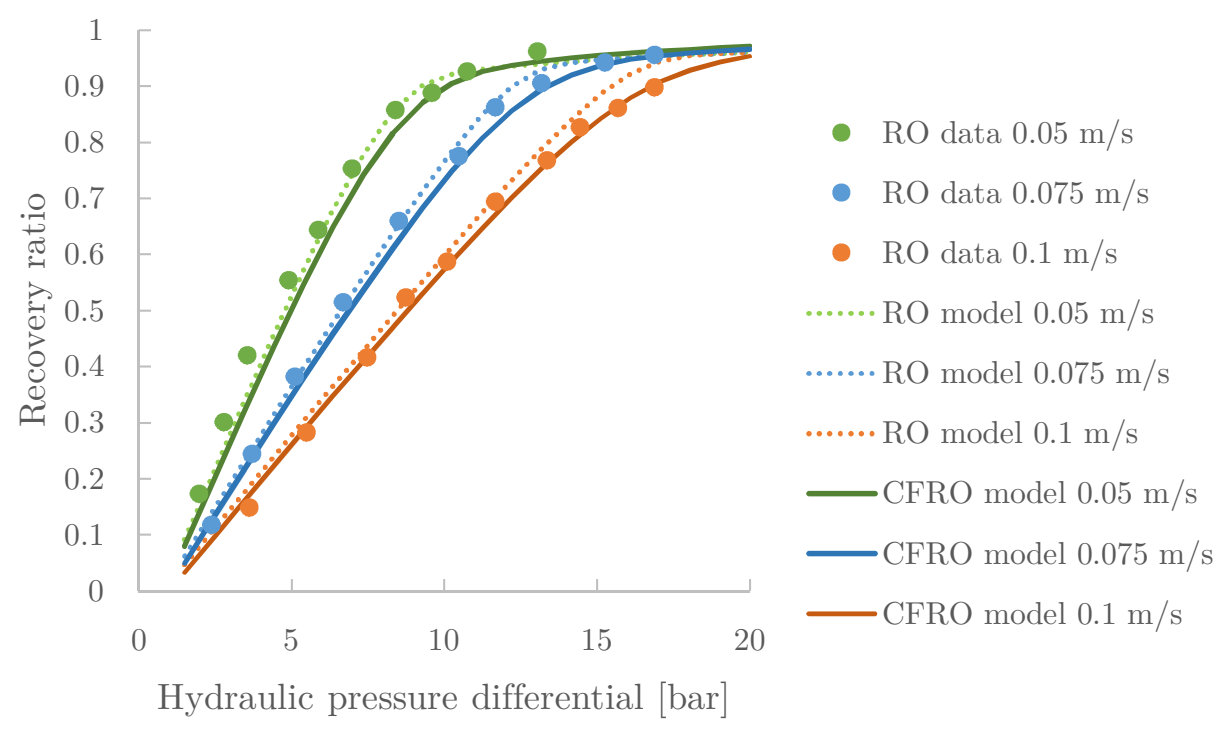

Figure 2-4: Comparison of data from $\mathrm{RO}$ experiments by Song et al. and the model presented in this paper. 
The results of our simulations plotted against the results from the published articles are shown in Figures 2-3 and 2-4. Good agreement with both sets of simulations is observed.

\subsection{Optimization schemes}

In several parts of this thesis, we seek to recommend design conditions for CFRO systems. In order to do this, we wish to find the best performing system with regard to $\eta_{I I}, S E C, R R$, or some other performance metric. We use two different optimization schemes built into EES to determine optimal system design parameters. The first, the genetic method, is complicated and highly computationally expensive, but provides high accuracy and reduces the risk of finding local maxima or minima instead of global values, in cases where both exist [41]. When optimizing many values of a single variable, performing many genetic optimizations is too computationally expensive. Instead, the conjugate directions method, a gradient-based method is used. Because there is a chance that local optima are found with the conjugate directions method, the points optimized with the genetic method serve as a check that the true maximum or minimum is being found. 


\section{Chapter 3}

\section{Single Module Analysis}

\subsection{Base case}

As a baseline, we simulate a single CFRO stage processing brine at a concentration of $70 \mathrm{~g} / \mathrm{kg}$, a typical concentration for RO brine. Initially, we choose a membrane area of $150 \mathrm{~m}^{2}$, roughly the size of four standard $20 \mathrm{~cm}$ spiral-wound $\mathrm{RO}$ elements. The fluid velocity at the concentrate side inlet is set to $10 \mathrm{~cm} / \mathrm{s}$, while the fluid velocity at the diluate side inlet is set to $5 \mathrm{~cm} / \mathrm{s}$. These numbers are chosen as representative values that could be found inside a normal RO system. Significantly higher velocities will lead to more drag inside the system and require higher fluxes and pressures to achieve the same outlet conditions, while significantly lower velocities will result in a very high rate of fouling [42] and concentration polarization. One last variable is required to fix the system, which can be a recovery ratio, outlet salinity, or applied pressure. We choose a recovery ratio of $20 \%$ to be the baseline condition, where the recovery ratio is given by Equation 1.3

The base case system takes in $1.77 \mathrm{~kg} / \mathrm{s}$ of feed water at a concentration of 70 $\mathrm{g} / \mathrm{kg}$, with mass flow rates of $1.18 \mathrm{~kg} / \mathrm{s}$ at the concentrate inlet and $0.59 \mathrm{~kg} / \mathrm{s}$ at the diluate inlet. A hydraulic pressure of 50 bar is applied in order to recover $20 \%$ of the feed, resulting in a concentrate stream at a flow rate of $0.94 \mathrm{~kg} / \mathrm{s}$ at a concentration of $87 \mathrm{~g} / \mathrm{kg}$, and a diluate stream at a flow rate of $0.82 \mathrm{~kg} / \mathrm{s}$ at a concentration of $51 \mathrm{~g} / \mathrm{kg}$. The specific energy consumption for this system is $2.1 \mathrm{kWh}$ used per $\mathrm{m}^{3}$ 
of solution that passes through the membrane. The resulting $2^{\text {nd }}$ law efficiency, as defined in Section 1.3.3, is 22.7\%. Although this recovery ratio seems low, an RO system operating at the same applied pressure would not be able to recover any water, as the osmotic pressure at the inlet to the system is 60.5 bar.

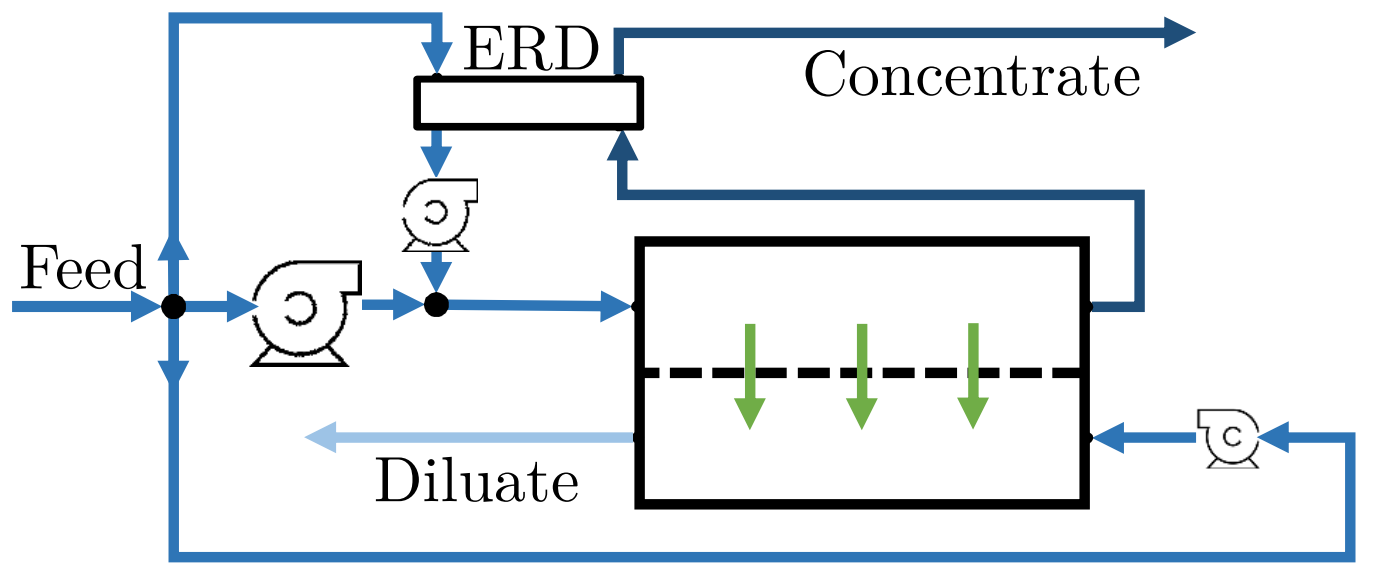

Figure 3-1: Diagram of a single split-feed CFRO stage, showing the high pressure and booster pumps to the left of the membrane, and the circulation pump to the right, as well as the energy recovery device (ERD). The color intensity of the blue arrows correlates with stream solution concentration, while the green arrows show the direction of permeate flow.

The setup for this system is the same as is shown in Figure 3-1, and the results of the base case simulation are displayed in Figure 3-2. In Figure 3-2, the osmotic pressures at five points in the module are shown, along with the flow direction at each point. The $\Delta \pi_{b u l k}$ label shows the $\Delta \pi$ between the two bulk flows, and is the osmotic pressure that would need to be overcome in order to produce water flux through the membrane in an ideal case when there are no losses in the system. However, due to the effects of concentration polarization, which includes internal concentration polarization $\left(\Delta \pi_{I C P}\right)$ and external concentration polarization on both sides of the membrane, the actual osmotic pressure difference at the membrane active layer, $\Delta \pi_{\text {actual }}$, is much larger. Concentration polarization is discussed in detail in Section 2.3, as well as in various sources in the literature [35, 36, 43]. Although not shown in Figure 3-2, there are additional losses in the system, including head loss due to viscous drag in the module and inefficiencies in system components such as pumps and energy recovery devices (ERD's). 


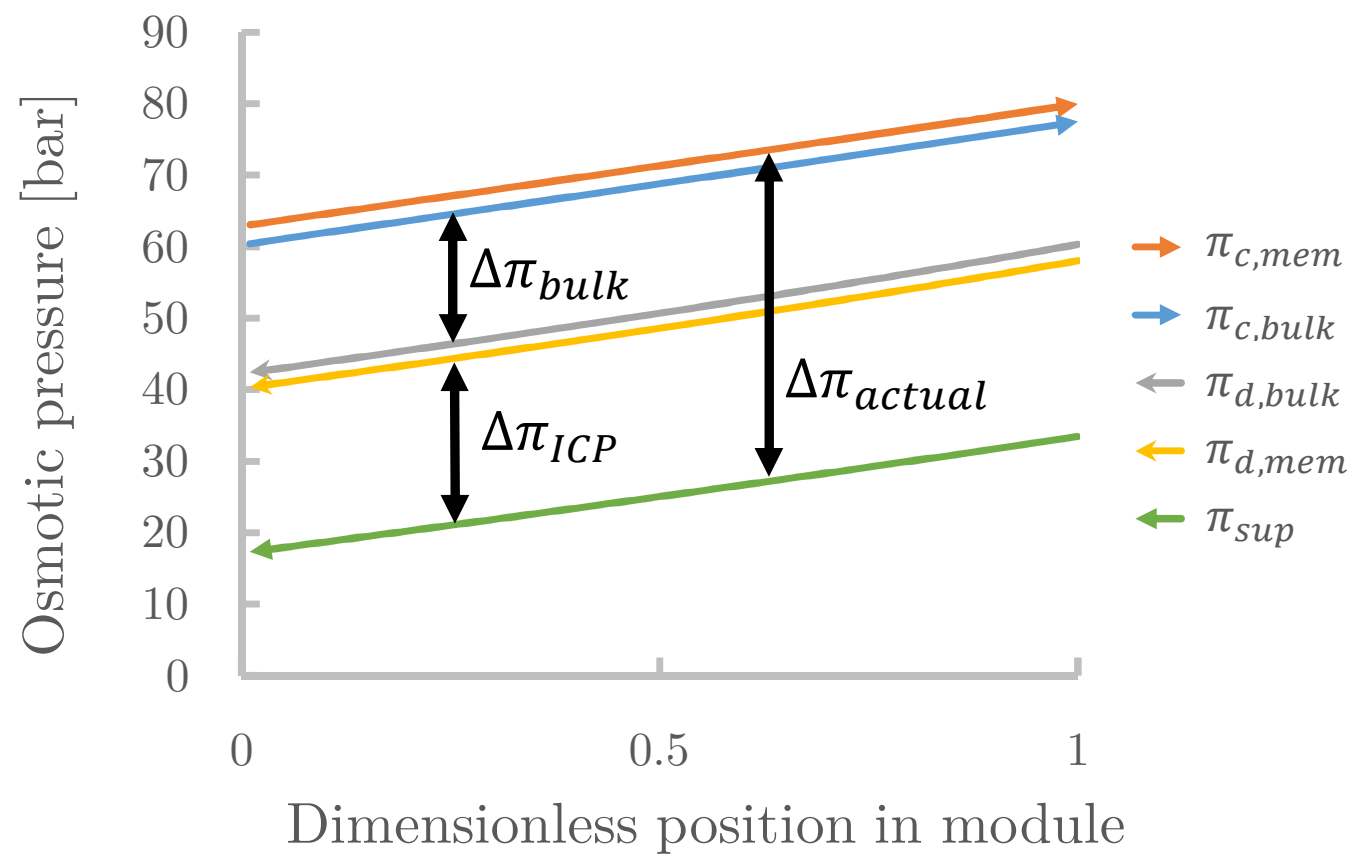

Figure 3-2: Osmotic pressures shown along the length of the membrane in a single CFRO stage during base-case operation.

\subsection{Parametric analysis}

Modifying the key parameters of this system allow us to better understand system operation. The parameters investigated in Section 3.2.1 include the membrane area and recovery ratio. The effects of varying membrane characteristics are examined in Section 3.2.2.

\subsubsection{System parameters}

\section{Flux}

The membrane area of the system is linked directly to the membrane flux, and varying this parameter significantly changes the performance of the system. Reducing the membrane area while keeping the same inlet and outlet conditions will require an increase in flux and an increase in applied pressure, resulting in increased energy consumption and increased operating costs. However, reduced membrane areas will also reduce the overall size and capital costs of the system. The results of the parametric analysis with varying membrane area are shown in Figure 3-3. 
Operating at high fluxes can greatly increase the losses due to concentration polarization, and requires an increase in the applied pressure to achieve the desired flux. While external concentration polarization (ECP) is a function of flux, at low fluxes its contribution to the system losses is much smaller than that of internal concentration polarization (ICP). This is largely due to the fact that the thickness of the concentration boundary layers for ECP is very small, on the order of 30 microns for the base case described above, while the effective thickness over which ICP acts is given by the membrane's structural parameter, which is more than an order of magnitude larger than the external concentration boundary layers. Because solutes must diffuse through a layer with a much larger effective thickness, the change in osmotic pressure across the ICP layer is very large. However, as the membrane flux increases, the rate of salt advection increases, resulting in a thicker concentration boundary layer and greater losses due to ECP.

As Figure 3-3 shows, operation at high flux is inefficient due to the large losses caused by concentration polarization. At lower fluxes, around $1.5 \mathrm{~L} / \mathrm{m}^{2}-\mathrm{h}$, very effi-

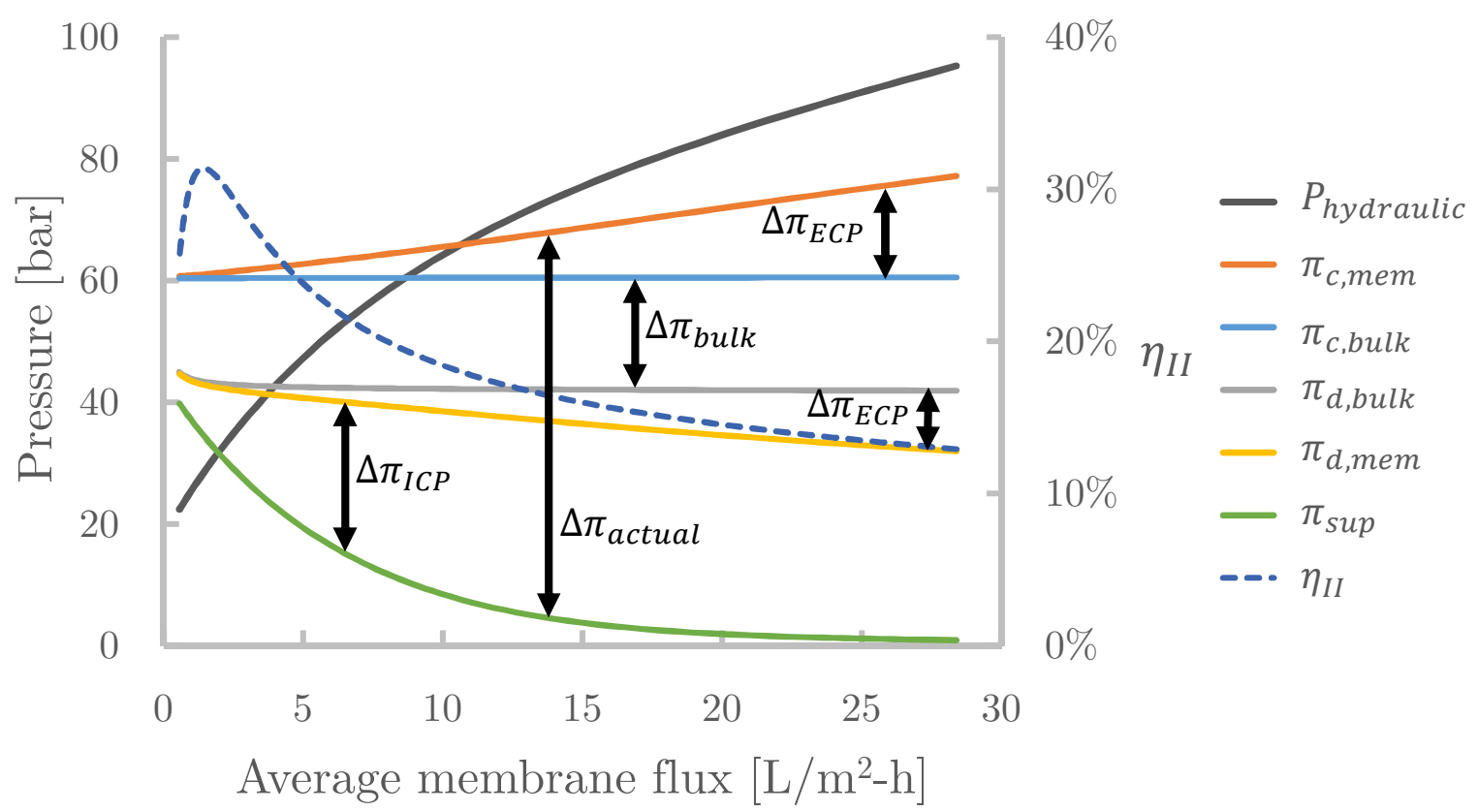

Figure 3-3: Osmotic pressures taken at one location in the module, the concentrate inlet, calculated at various membrane sizes, resulting in varying membrane flux. Inlet conditions and recovery ratio remain fixed in this case. The hydraulic pressure and $2^{\text {nd }}$ law efficiency are also shown. 
cient operation is possible, but potentially very expensive due to the large membrane area required. For reference, most seawater RO plants operate at an average membrane flux of approximately $15 \mathrm{~L} / \mathrm{m}^{2}-\mathrm{h}$ [39]. Interestingly, if the membrane area continues to grow and flux drops below $1.5 \mathrm{~L} / \mathrm{m}^{2}-\mathrm{h}$, the $2^{\text {nd }}$ law efficiency decreases again. In this region, the losses due to concentration polarization effects are minimal, but large amounts of salt diffusion across the very large membrane actually increase the diluate outlet salinity and reduce the concentrate outlet salinity. This salt flux results in less useful work being done, even though the amount of water that flows through the membrane is the same, resulting in a reduced $2^{\text {nd }}$ law efficiency. Additionally, at very large membrane areas, losses due to drag become large, increasing the energy consumed by the system while not affecting the amount of useful work done. Therefore, for any given operating conditions, there exists a membrane size that will minimize the energy consumption of the system by balancing the losses due to concentration polarization, salt diffusion, and drag. In the case of an ideal membrane with perfect salt rejection, the membrane size corresponding to the minimum energy consumption is determined by balancing the losses due to concentration polarization and drag.

\section{Recovery ratio}

Varying the recovery ratio while the membrane dimensions and feed conditions are fixed is accomplished by varying the applied hydraulic pressure, which increases the flux and the total amount of permeate. Figure 3-4 shows how the outlet concentrations vary as the recovery ratio increases, as well as the $2^{\text {nd }}$ law efficiency over the same range. Interestingly, the highest $2^{\text {nd }}$ law efficiency in this case is not attained at very low fluxes, as was the case for a system with fixed recovery. In order to better understand what is going on in this case, we calculate exergy destruction and thermodynamic least work for each recovery ratio, and break the exergy destruction down into its different constituent parts in order to gain insights into where inefficiency occurs. 


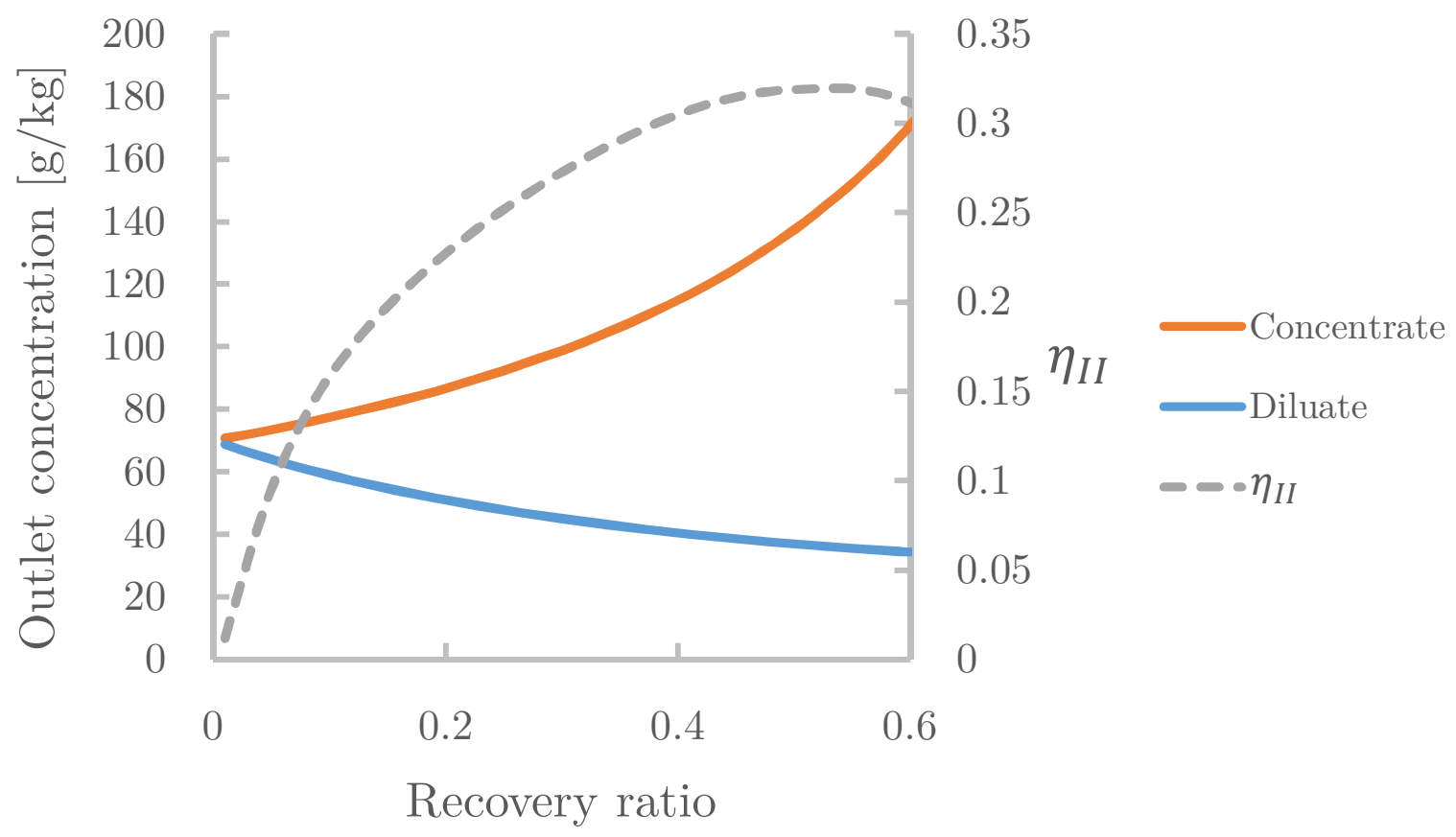

Figure 3-4: Outlet concentrations and $2^{\text {nd }}$ law efficiency shown over a range of recovery ratios.

\section{Exergy destruction}

The exergy destruction for various components in the system is calculated using the methods described by Mistry et al. [12] for entropy generation mechanisms in desalination system components. The results of this calculation are shown in Figures 3-5 and 3-6. Exergy destruction or entropy generation occurs due to losses in the ERD and pumps, head losses due to drag in the membrane module, pressure drop across the membrane, and the mixing of streams of different salinities as the permeated water and salt mix with the diluate stream. The pressure drop across the membrane can be separated into the useful work done and the losses due to concentration polarization and membrane resistance. The sum of the useful work done plus all the exergy destruction in the system will sum to the total work input into the system.

By looking at exergy destruction throughout the system, we can see that for a fixed system, exergy destruction due to drag remains relatively constant no matter what the recovery ratio is. This is rather intuitive, as the same flow rates enter the system at the same velocity regardless of recovery ratio. While increasing the 


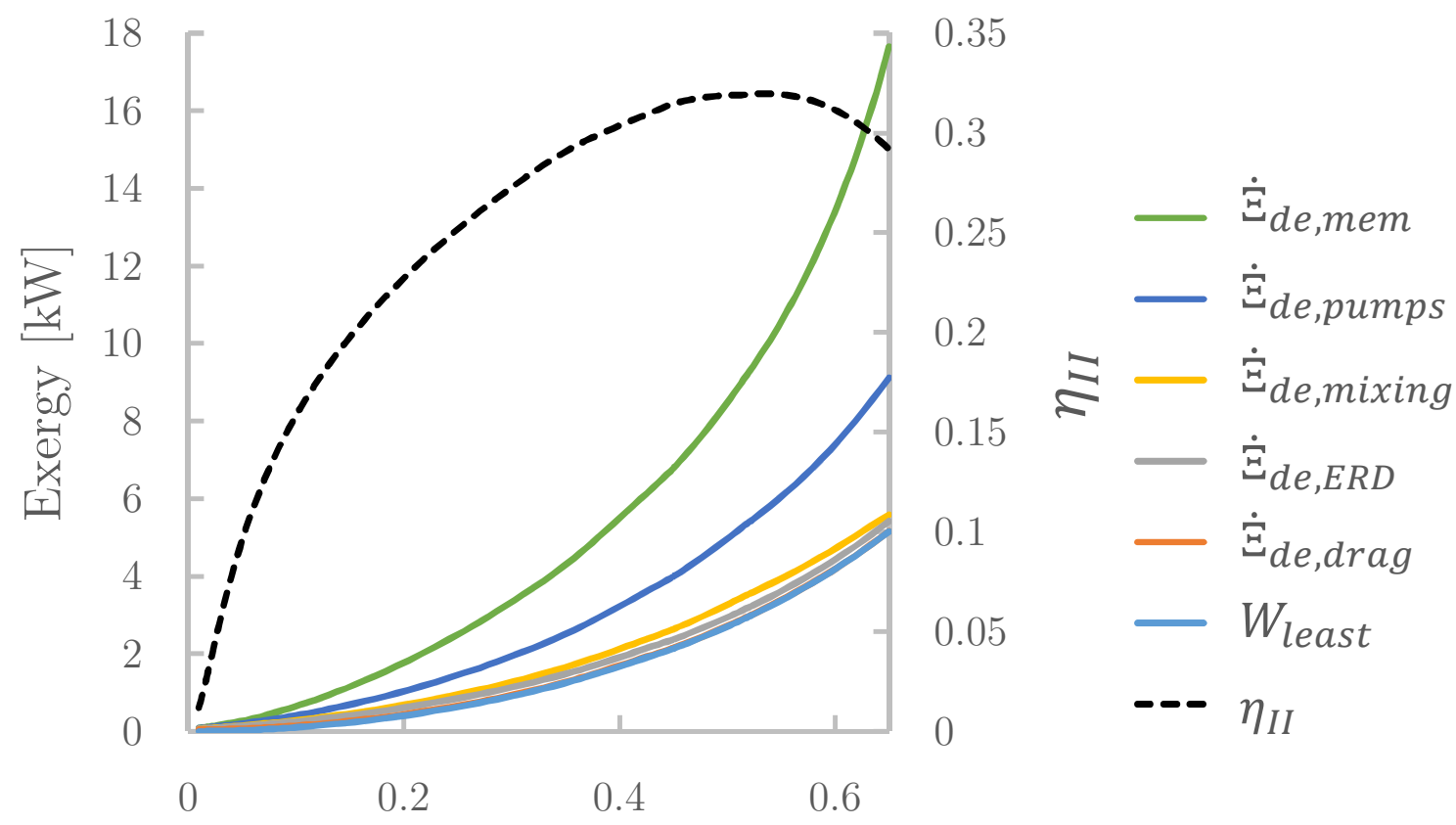

Recovery ratio

Figure 3-5: Stacked line graph showing the work into a CFRO system as a function of recovery ratio, broken down into the useful work performed and exergy destruction within different components in the system.

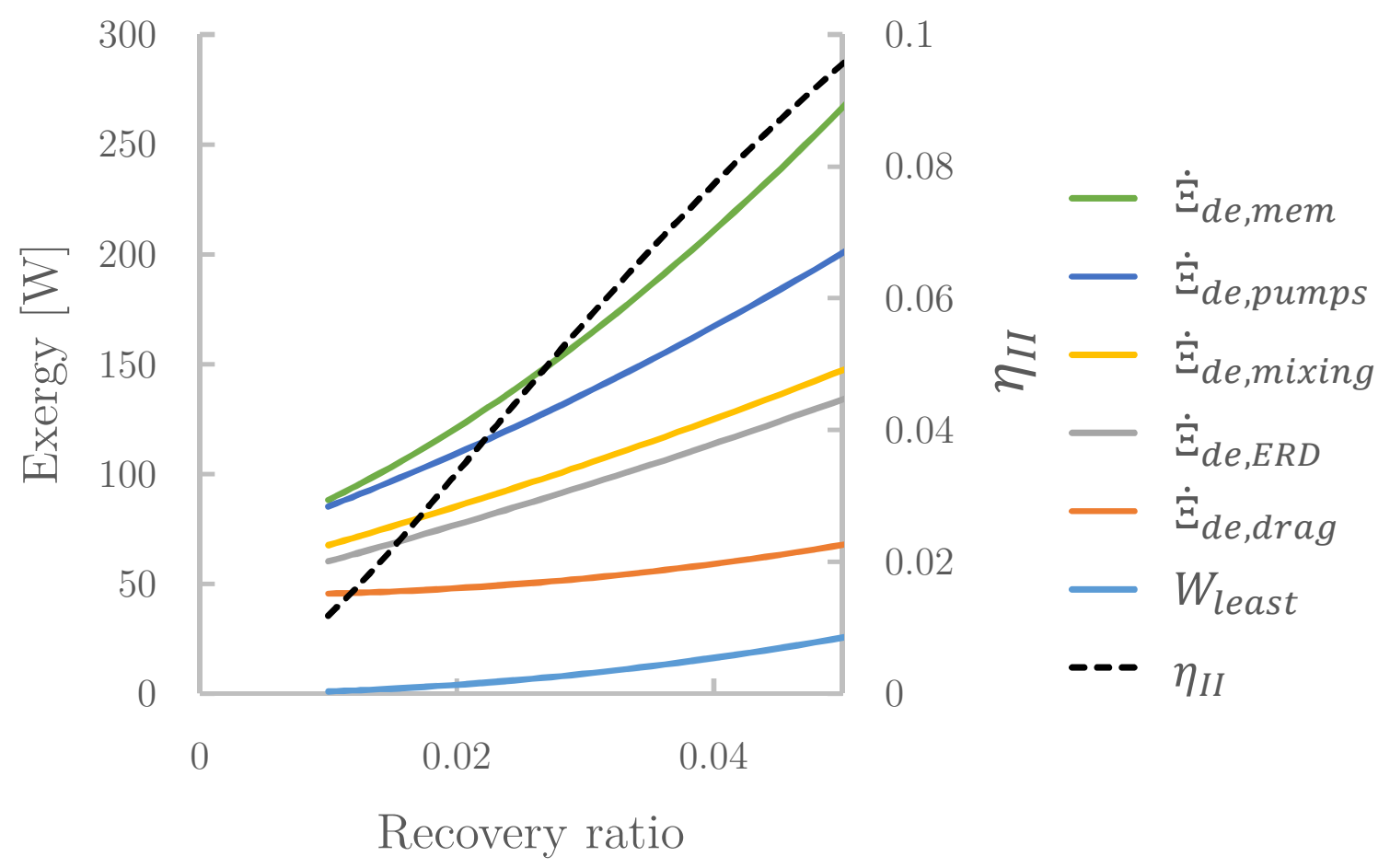

Figure 3-6: Stacked line graph showing the work into a CFRO system as a function of recovery ratio, shown at very low recovery ratios, broken down into the useful work performed and exergy destruction within different components in the system. 
recovery ratio will lead to lower velocities and less drag on the concentrate side, the velocities and drag will increase on the diluate side, with the net effect of maintaining relatively constant losses due to drag. Other sources of entropy generation or exergy destruction scale with the recovery ratio. This means that, at very low recovery ratios, when the useful work done approaches zero, but losses due to drag remain constant, the resulting $2^{\text {nd }}$ law efficiency is will also approach zero. As the recovery ratio increases, drag losses become negligible, and the $2^{\text {nd }}$ law efficiency increases. At very high recovery ratios, when the flux becomes very high, the losses due to concentration polarization increase more rapidly than the useful work done, forcing the $2^{\text {nd }}$ law efficiency to decrease again.

\section{Equipartitioning of entropy generation}

The exergy destruction analysis also shows that the exergy destruction in the membrane and pumps are the largest sources of losses in the system. The exergy destruction in these two components are linked, as they are both a function of the pressures within the system. The losses in the membrane are the result of depressurization of the liquid that does not accomplish useful work, which can occur because of the membrane's resistance to flow or due to concentration polarization. The losses in the pump are due to the inefficiency of the pump itself, the volumetric flow rate, and the applied pressure. Several methods for improving the efficiency of the system and reducing entropy generation include increasing the pump component efficiency, reducing the resistance of the membrane by increasing its permeability, reducing the effects of ICP by modifying membrane parameters, and reducing the required pressure by reducing the membrane flux. The applied pressure can also be reduced by reducing variations in flux along the length of the membrane.

This last method of reducing entropy generation is also known as balancing the system or equipartitioning the entropy generation in the stage. According to the theory of thermodynamic equipartitioning, the best configuration of a mass exchanger is that in which the entropy production rate is most uniformly distributed [44]. According to Thiel et al., this uniform distribution, or equipartition, of entropy production occurs 
when the thermodynamic driving force is equipartitioned for processes that have a constant force-flux coefficient, such as solution diffusion through a semi-permeable membrane, as seen in RO and CFRO systems [45]. In this case, the thermodynamic driving force is $\Delta P-\Delta \pi$, and the system will be balanced when this quantity, which is directly proportional to the flux, is balanced throughout the module.

However, because entropy generation results from several different phenomena, not just the irreversible depressurization of a liquid, the system as a whole may be optimized even when the entropy generation within each membrane module is not equipartitioned. This is especially true for multistaged systems. Generally, the benefit of multistaging desalination systems, such as ED [46] and CFRO, is that it allows us to equipartition the entropy generation over the length of the system. However, if significant entropy generation occurs in the additional equipment required to multistage, such as extra pumps and ERD's, or if the imbalance in driving force is small relative to the driving force itself, performance may actually be improved by not multistaging. This will depend on the specific system in question, its operating parameters, and the efficiency of the system's components.

\subsubsection{Membrane parameters}

Internal concentration polarization results in an enormous increase in required applied pressure, and so limiting this effect should be one of the main goals of membrane development for CFRO-specific membranes. The equations governing concentration polarization, shown in Equations 2.8 through 2.10, show that the change in concentration due to ICP is dependent on the membrane's structural parameter and salt permeability coefficient, the water flux, and the diffusion coefficient. While the diffusion coefficient of salt in water is not something that can be changed, the salt permeability coefficient, water permeability coefficient, and structural parameter are. 


\section{Membrane salt permeability B}

First we examine what happens if we change the membrane salt permeability coefficient $B$. To analyze this value, we look at a single exchanger with similar conditions as described in Section 3, with the only modification being that a fixed pressure of 70 bar is applied instead of fixing the recovery ratio. The $B$ coefficient is varied from 0.1 to 10 and the results are shown in Figure 3-7, but the results are inconclusive as to whether low or high $B$ values are more beneficial.

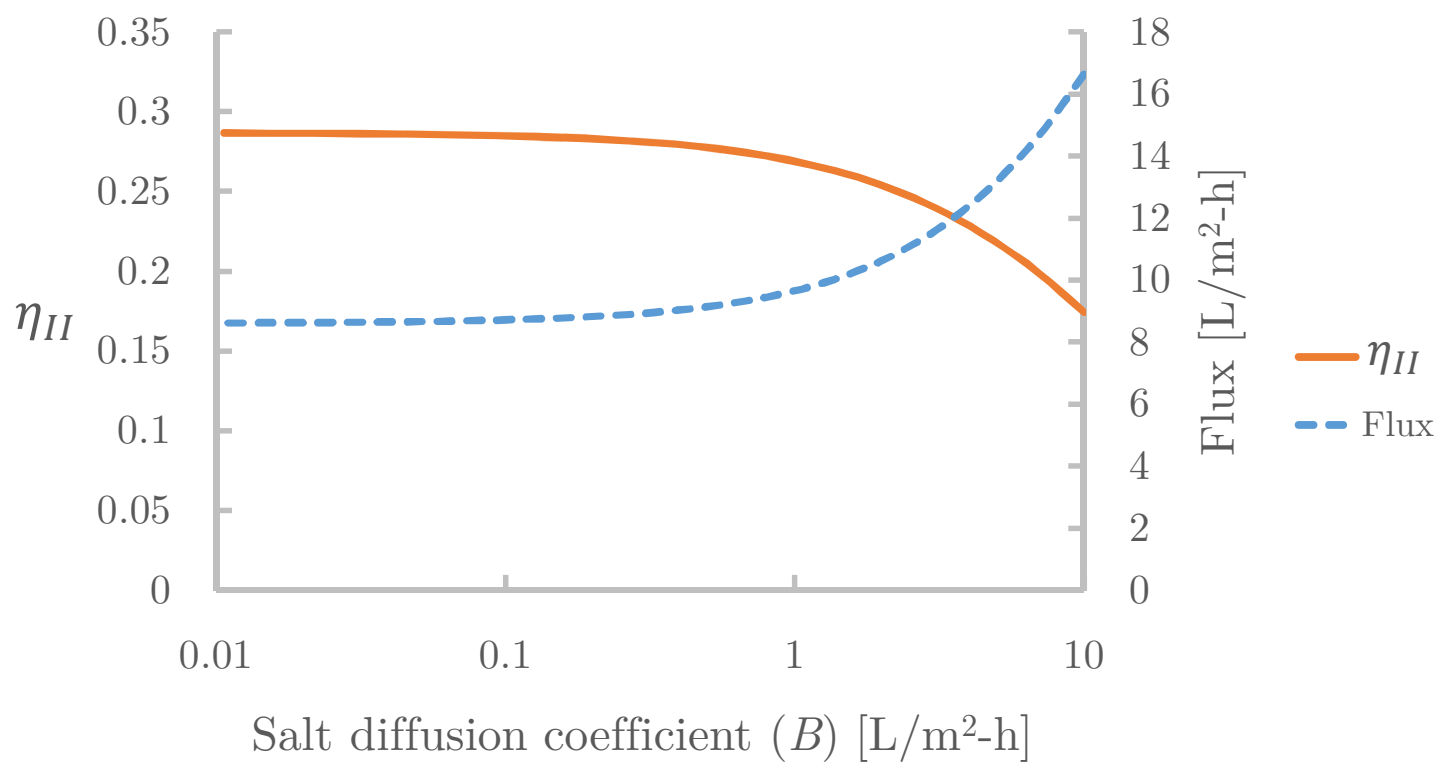

Figure 3-7: As the salt diffusion coefficient $B$ increases, the CFRO system's efficiency decreases, but the flux in the system increases due to the reduced internal concentration polarization.

There are several reasons that high $B$ values, or "leaky" membranes may be beneficial. With a fixed hydraulic pressure and fixed inlet conditions, a high $B$ value membrane allows salt to flow more easily, increasing the concentration at the interface between the active and support layers of the membrane and reducing the effects of ICP. The $\Delta \pi$ across the active layer of the membrane is reduced, allowing for greater fluxes. Increased fluxes may allow for reduced membrane areas and reduced capital costs. Another potential benefit is that, for the conditions examined here, the concentrate stream's outlet concentration increases, which may allow for fewer stages to accomplish the same desired increase in concentration of the original feed 
stream. This increase in concentration is also due to the increased flux, which helps to de-water the concentrate stream.

On the other hand, leaky membranes may be unhelpful for several reasons. The

$2^{\text {nd }}$ law efficiency, as shown in Figure 3-7, always decreases with increasing $B$ value, because the additional salt leaking through the membrane reduces the useful work done by the system. So, while the specific energy consumption is reduced for high $B$ values, the useful work done drops as well, resulting in a reduced $2^{\text {nd }}$ law efficiency. It is important to note that changing the $B$ value under these conditions results in a change in outlet conditions, and thus a change in the systems being compared. To avoid comparing different systems, this analysis is continued with a multi-module configuration in Section 6.2.

\section{Pure water permeability A}

Now we examine how the membrane pure water permeability coefficient, $A$, changes the performance of a CFRO system. For this analysis, the same parameters are used as in Section 3.2.2, with the exception of using a fixed recovery ratio of $20 \%$ instead of a fixed pressure to constrain the system. While holding $B$ constant and varying $A$, we can see in Figure 3-8 that an increased $A$ increases the flux through the system and allows for more efficient operation because of reduced membrane resistance. However, as predicted by McGovern et al. [47], if all other parameters are held constant, increasing $A$ will allow the flux to increase asymptotically, until the membrane resistance is negligible compared to the mass transfer resistances that are a result of concentration polarization. As Figure 3-8 shows, there are diminishing returns for this specific case when the permeability increases above approximately 2 $\mathrm{L} / \mathrm{m}^{2}$-h-bar.

\section{Structural parameter $\mathbf{S}$}

The structural parameter $S$ is one of the main factors governing ICP, as discussed in Section 2.3. By reducing this parameter, it becomes easier for salt to diffuse through the support layer towards the membrane's active layer interface, and the $\Delta \pi$ across 


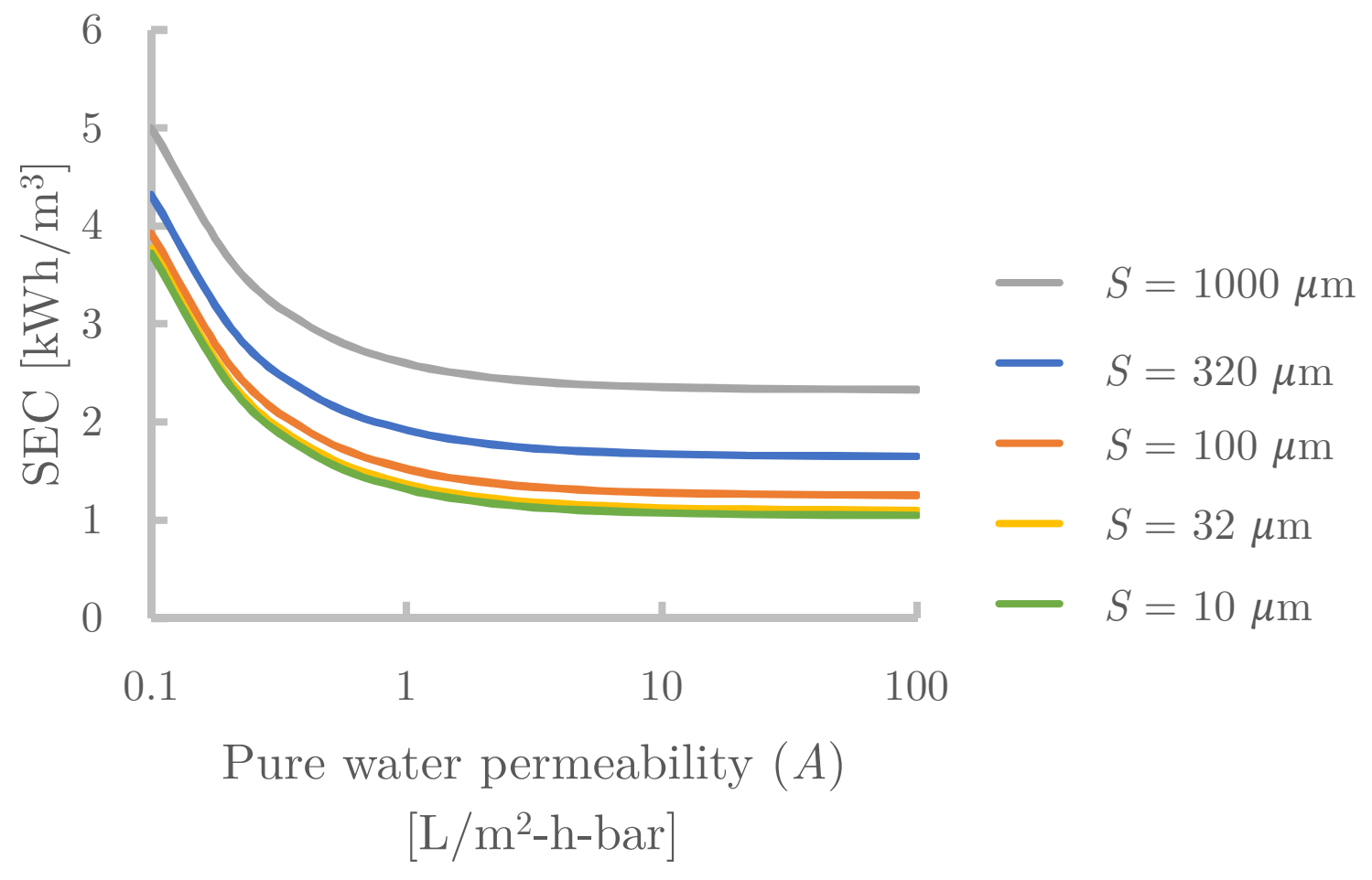

Figure 3-8: The specific energy consumption (SEC) is reduced as the pure water permeability $A$ increases or the membrane structural parameter $S$ decreases. Asymptotic behavior is experienced when other mass transfer resistances in the system begin to dominate. 
the membrane is reduced, allowing for greater fluxes and reduced losses. As for the $A$ parameter, diminishing returns accompany a reduction of the structural parameter, and the specific energy consumption decreases asymptotically with decreasing $S$, as shown in Figure 3-8. Once the structural parameter is sufficiently small, resistance and losses due to ICP are negligible compared to the resistance to mass transfer caused by ECP, and further reductions in the structural parameter no longer noticeably improve performance. 
THIS PAGE INTENTIONALLY LEFT BLANK 


\section{Chapter 4}

\section{CFRO Configurations}

CFRO is a young technology that has undergone limited development. The academic work is limited to what has been done by Bartholomew et al. [22] and Chen et al. [23]. Both use the same core CFRO technology, but organize modules or stages into different configurations to accomplish different goals. For clarity, in this thesis we will refer to the core technology itself, a membrane-based, counterflow, osmotic mass exchanger, as counterflow reverse osmosis (CFRO). We refer to the configuration introduced by Bartholomew et al. as consecutive loop CFRO and the configuration introduced by Chen et al. as feed-through CFRO. In Section 4.3, we introduce a third configuration, which we will call split feed CFRO. These three configurations are discussed below.

\subsection{Consecutive loop CFRO}

With the consecutive loop (or OARO) configuration introduced by Bartholomew et al. and shown in Figure 4-1, the authors show potential to recover water from high salinity brines, such as produced water from natural gas fracking operations, much more efficiently than a comparable MVC system [22]. While their presented results are very promising, significant operational difficulties remain to be addressed.

One simplification made in the modeling work of [22] was to neglect salt flux through the membranes. While the amount of salt flux through the membranes 
may be of a negligible value, the phenomena is important to the salt balance of the loops. In this configuration, permeate flows from one recirculating loop into the next, continuing until the permeate exits the system as RO permeate. In order to maintain steady state operation, the amount of water and salt flowing into each loop must be equivalent to the amount of water and salt flowing out of the loop. If salt flow rates are not equal, salt will either accumulate or be depleted within a loop, which would negatively affect the performance of the system and possibly prevent the system from functioning altogether.

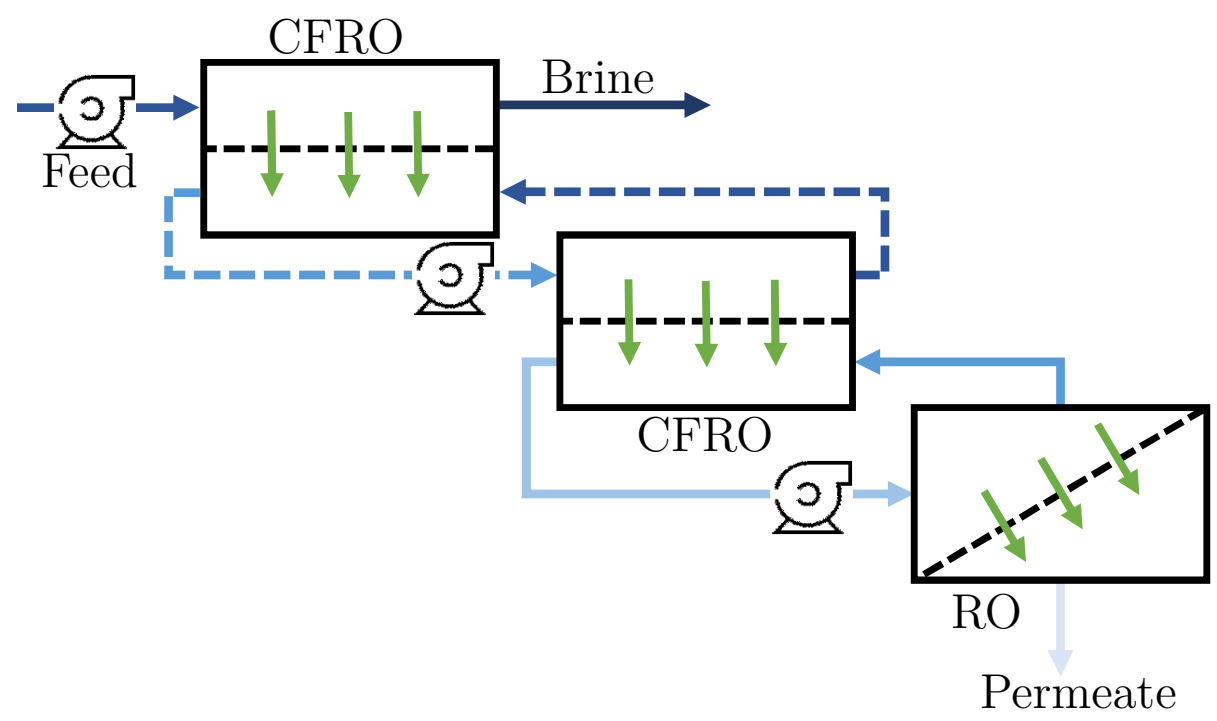

Figure 4-1: Diagram of a simple consecutive loop configuration with two CFRO stages and an RO stage. The dashed stream lines show the path of a single loop. The color intensity of the blue arrows correlates with stream solution concentration, while the green arrows show the direction of permeate flow.

While water flux through each membrane can be modified to achieve steady state operation by modifying the applied hydraulic pressure, maintaining constant salt flux into and out of each loop is much more difficult. One way to maintain stable salt flux in the face of fluctuating feed conditions would be to employ an additional salt makeup stream or a pure water stream that mixes with the solution in each loop in order to counter the effects of imbalanced salt flow and achieve system stability. However, mixing streams of different concentrations would result in additional entropy generation and a less efficient system. While this challenge is by no means insurmountable, it adds complexity to operation of this CFRO configuration. 
In addition, the recovery ratio may face practical limitations. In order to achieve high recovery ratios, either high fluxes or very large membrane areas will be required. In order to maintain steady state conditions, the large membrane permeate volumes will have to be equivalent in each loop in the system. Large membrane areas lead to high capital costs, and high fluxes can lead to reduced energy efficiency and higher operating costs.

One potential advantage of this system relative to other CFRO configurations is that the solution recirculating in each closed loop does not necessarily have to be of the same composition as the feed stream. While using the same basic feed solution in all stages may simplify the operation, using different solutions could hold potential operational advantages with regard to fouling and scaling.

\section{$4.2 \quad$ Feed-through CFRO}

Chen et al. show the potential of another configuration, feed-through CFRO (or COMRO), to improve on available brine concentration methods in several regards: increasing the maximum salinity that can be treated with a pressure-driven membrane desalination processes, increasing the maximum recovery ratio of lower salinity feed systems, increasing energy efficiency, and reducing operating pressures. Feed-through CFRO uses one or more CFRO stages to dilute an RO feed stream by extracting water from RO brine. A diagram of this system is shown in Figure 4-2. For an in depth description and analysis of the operation of this system, see Chen et al. [23].

By reducing the concentration at the $\mathrm{RO}$ inlet and increasing the volume, the RO stage is able to extract more permeate without increasing the pressure, thus increasing the recovery ratio of the system. However, depending on feed conditions and membrane parameters, very large increases in recovery ratio may not be achievable. The maximum recovery ratio of a feed-through CFRO configuration is determined by the burst pressure of the membrane and the feed concentration. When the osmotic pressure difference between the exiting brine stream and the feed stream, $\Delta \pi$, is equal

to the maximum hydraulic pressure allowed in the system, $\Delta P_{\max }$, no more permeate 


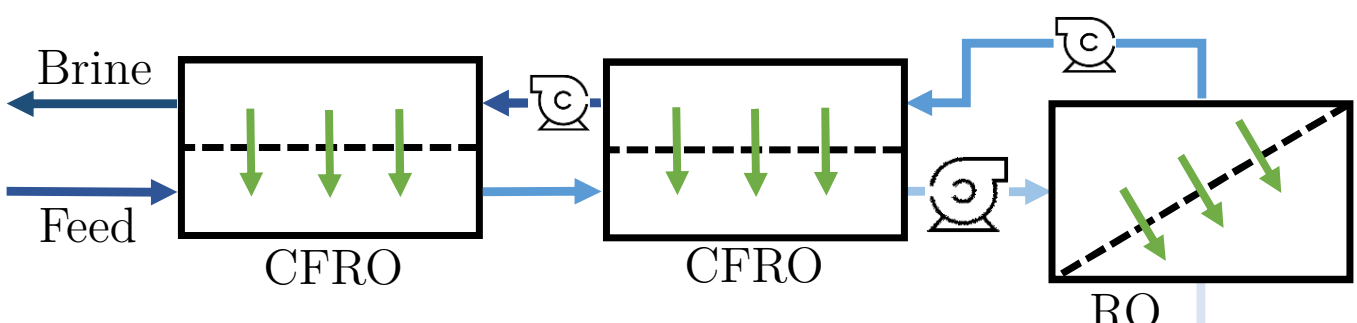

Permeate

Figure 4-2: Diagram of a simple feed-through CFRO configuration with two CFRO stages and one RO stage. The color intensity of the blue arrows correlates with stream solution concentration, while the green arrows show the direction of permeate flow.

can flow from the concentrate side of the membrane to the diluate side. As an example, for a system taking in seawater at $35 \mathrm{~g} / \mathrm{kg}$ and maximum hydraulic pressure of $70 \mathrm{bar}$, the maximum outlet salinity would correspond to a $\Delta \pi$ of 70 bar, with a resulting maximum brine salinity of approximately $110 \mathrm{~g} / \mathrm{kg}$. This limits the maximum recovery for the system to approximately $70 \%$. While this is an improvement over conventional RO, it remains well short of a ZLD system.

The effectiveness of this configuration may also be limited by its inherent imbalance, at least for the configuration shown. Because large volumes of water are removed from the system in the RO stage, the concentrate stream in the CFRO stages will have a much lower flow rate than the diluate stream. This imbalance in flow rates leads to imbalances in osmotic pressure differences across the membrane, and thus an imbalance in flux, as shown in Figure 4-3. At the side of the concentrate stream entrance, the osmotic pressure difference across the membrane is the lowest, and the flux is the highest. As the concentrate stream is dewatered, the concentration and osmotic pressure increase rapidly because the flow rate of water remaining on the concentrate side is small compared to the flow rate on the diluate side. The rapid increase in osmotic pressure results in rapidly declining flux. As was discussed in Section 3.2.1, imbalances in flux and driving force lead to unbalanced entropy generation in the system, which leaves room for improvements to the energy efficiency of the system. 


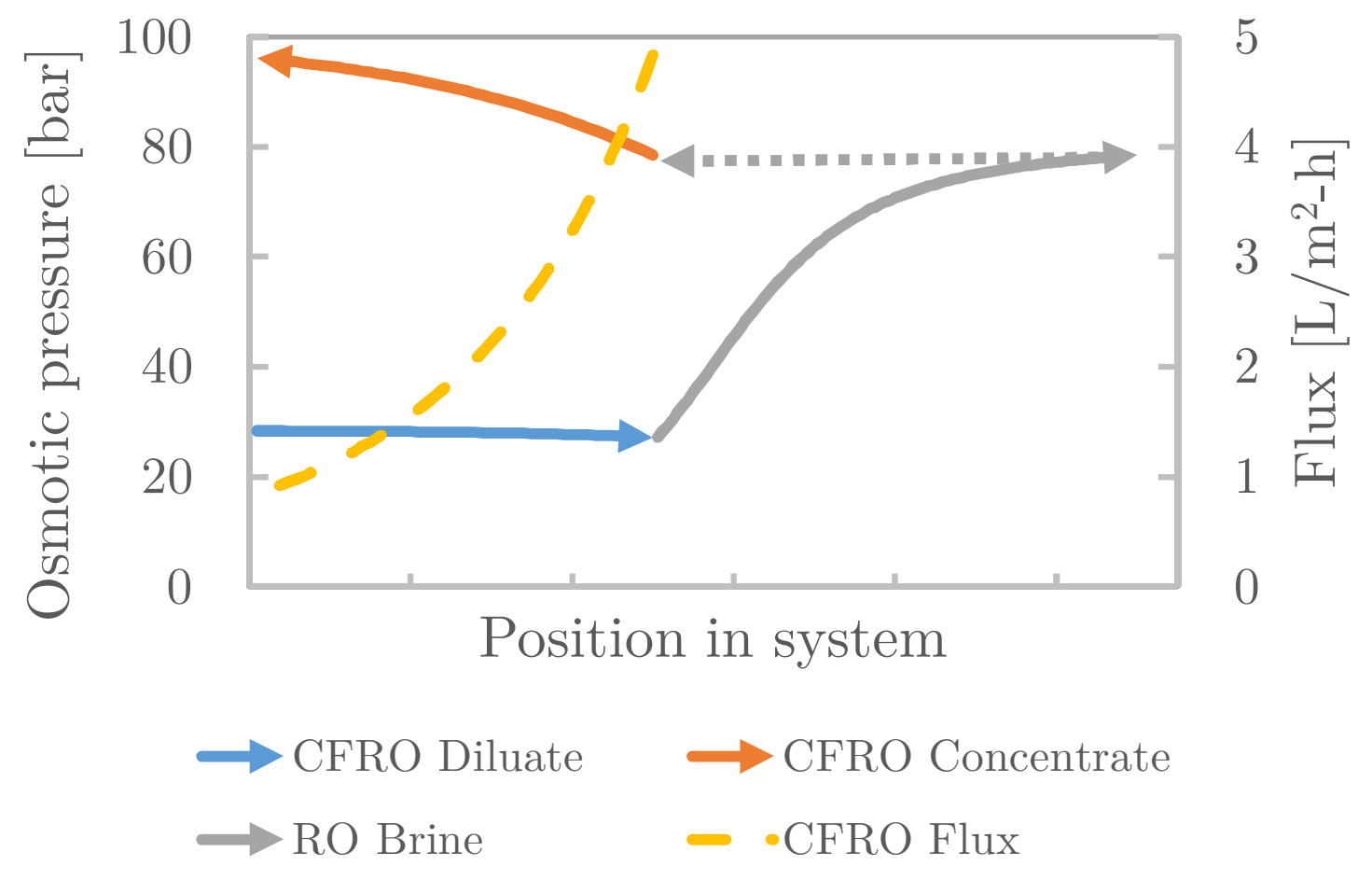

Figure 4-3: An unbalanced flux within CFRO stages is experienced in feed-through CFRO configurations due to unbalanced osmotic pressure changes on the two sides of the membrane, which arises because of imbalanced flow rates in the system.

\subsection{Split feed CFRO}

Split feed CFRO overcomes several of the challenges faced by feed-through and consecutive loop CFRO configurations. In a split feed configuration, shown in Figure 4-4, the feed stream entering each CFRO module is split into two streams, with one being pumped to high pressure and becoming the concentrate feed stream, while the other remains at low pressure and becomes the diluate feed stream. Because the two inlet streams are at the same osmotic pressure, any applied hydraulic pressure should generate some amount of flux through the membrane. This gives the system the ability to operate at very low hydraulic pressures.

In one realization of this configuration, Figure 4-4, the split feed CFRO modules can be hybridized with an RO module, with the lowest concentration diluate stream fed into the $\mathrm{RO}$ module, which produces purified water. If flow rates and pressures are selected carefully, the system can be set up in such a way that the RO concentrate stream is at the desired feed salinity for the first CFRO unit in the system, and 


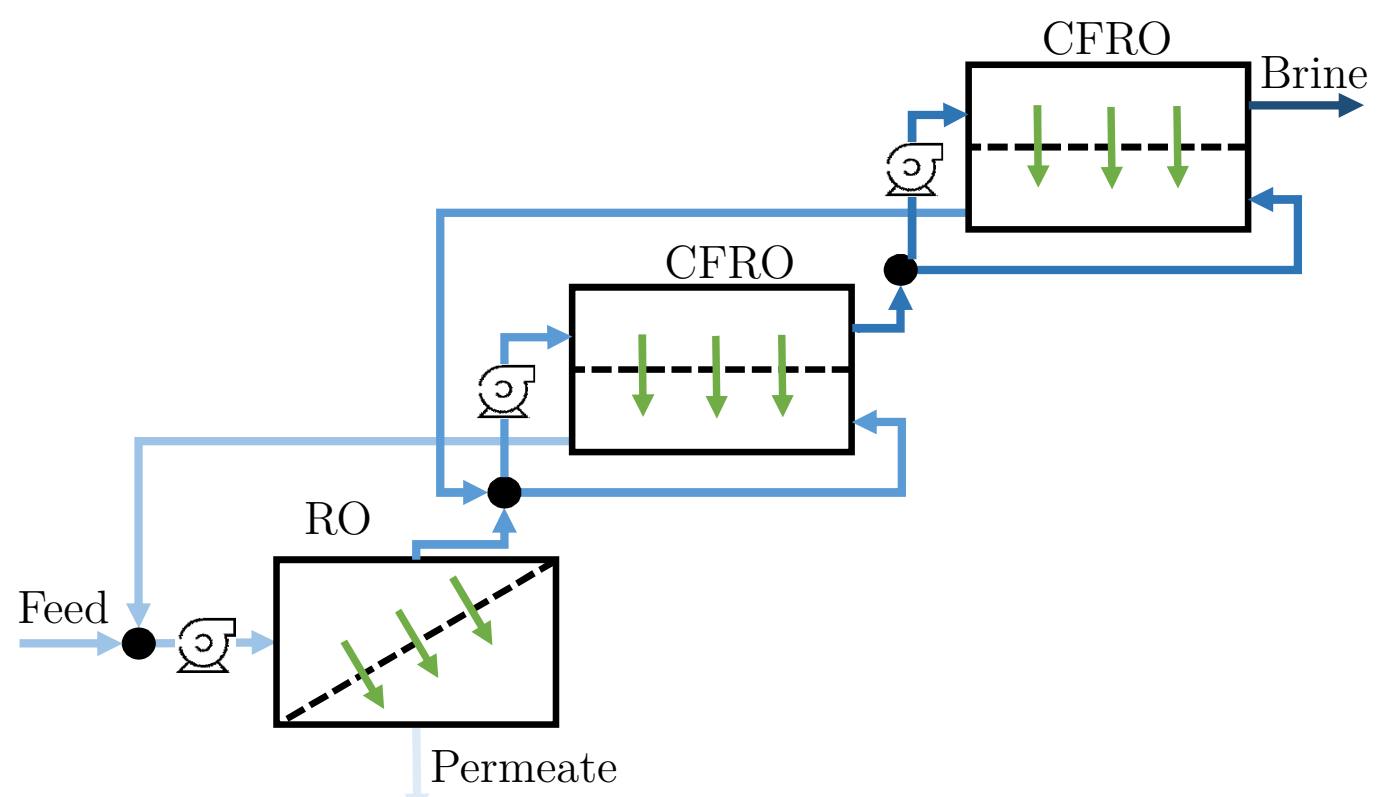

Figure 4-4: Diagram of a simple split feed CFRO configuration with two CFRO stages and a single RO stage. The color intensity of the blue arrows correlates with stream solution concentration, while the green arrows show the direction of permeate flow.

the CFRO diluate outlet is at the salinity of the RO inlet. This adjustment would avoid the losses inherent in directly mixing streams of different salinity. The system achieves higher recovery ratios than a standard RO system by recovering additional $\mathrm{RO}$ feedwater from the RO brine stream, so that the RO stage essentially treats a higher mass flow rate than is drawn from the source. This arrangement allows for either more water to be recovered from a given feed stream, or the same amount of water to be recovered from a smaller feed stream, reducing intake and pretreatment costs.

In both other configurations shown previously, the feed stream is restricted to entering the system at an extremity of the configuration. However, with the split feed system, the feed may instead be introduced at some midpoint within the system, as shown in Figure 4-5. Because of the additional degrees of freedom that arise from being able to control the flow rates on each side of the membrane, the feed stream can enter between two CFRO stages while maintaining flow to all points in the system, which allows for more freedom in system design. The additional degrees of freedom also allow for designs that achieve nearly any combination of feed 


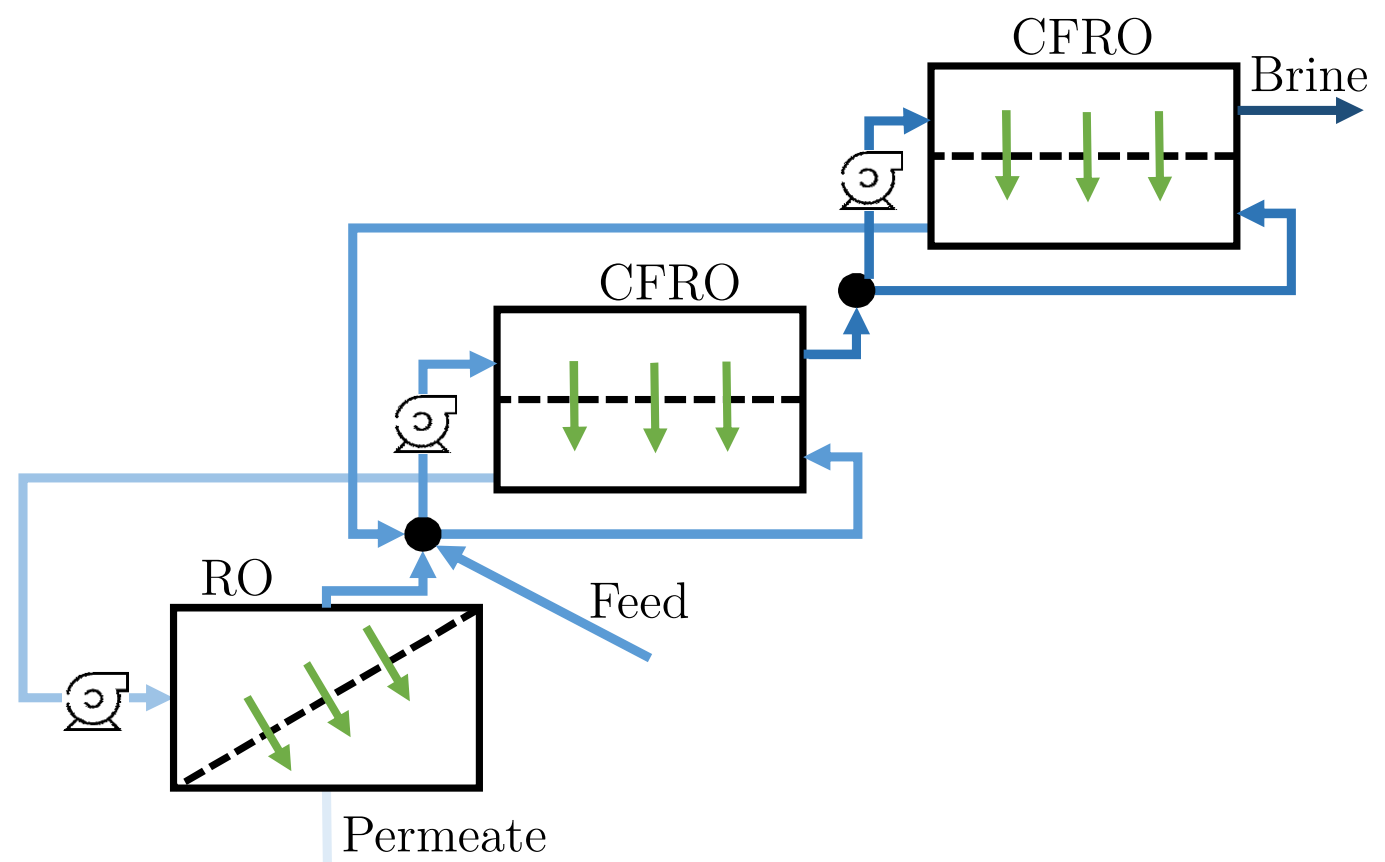

Figure 4-5: Diagram of a split feed CFRO configuration with a feed stream entering at a midpoint in the system.

and outlet concentrations, and allow for better balancing with respect to osmotic pressures and mass flow rates. The flows within the system can be managed so that the osmotic pressure difference across the membrane is relatively constant across the length of the module, which should result in more efficient operation from an entropy equipartitioning perspective, and which may lead to overall efficiency improvements.

While the recovery ratio is not limited in theory, certain practical factors that may limit the maximum solution concentration that can be produced by this configuration. As the saturation limits of the solution are approached, performance will likely degrade quickly due to membrane fouling and scaling, changes in membrane performance at high conentrations [39], and increased ICP effects at high concentrations. However, this technology may be able to generate brines of high enough concentration that evaporative crystallizers, solar evaporation ponds, or some other technology may be used to remove the last of the water from the solution, leaving valuable byproducts behind, eliminating liquid discharge, and providing new, environmentally friendly sources for minerals. 
Potential drawbacks of the split-feed configuration include the high capital costs due to the many stages that may be necessary for a high recovery operation, and complicated control systems to maintain system stability and performance. 


\section{Chapter 5}

\section{Split Feed CFRO Simulations}

\subsection{Setup}

We now simulate the performance of a split feed CFRO system. We consider a system with similar fixed conditions as in Section 3. The feed water has a mass flow rate of $1.25 \mathrm{~kg} / \mathrm{s}$ and a concentration of $35 \mathrm{~g} / \mathrm{kg}$. The total membrane area shared between the three stages is set to $450 \mathrm{~m}^{2}$. The final stage's outlet concentration, which sets the system's recovery ratio, is also fixed. Aside from these fixed parameters, 7 additional degrees of freedom need to be set in order for the system to be fully defined. These degrees of freedom include two variables which set the membrane area in each stage, three variables to set the membrane width in each stage, and two variables to set the two intermediate concentrations between the stages. The three variables that set the width of each stage also determine the inlet velocity for each stage. These seven variables are set by optimizing the system for minimum specific energy consumption using the methods described in Section 2.9.

This three-stage CFRO system is able to concentrate a $\mathrm{NaCl}$ solution with an initial concentration of $35 \mathrm{~g} / \mathrm{kg}$ up to $120 \mathrm{~g} / \mathrm{kg}$, for a resulting recovery ratio of $71 \%$. The system consumes $2.9 \mathrm{kWh}$ per cubic meter of RO permeate, with a resulting $2^{\text {nd }}$ law efficiency of $50.5 \%$. The osmotic pressure profile throughout the system is shown in Figure 5-1, and additional results are shown in Table 5.1.

These results show that the minimal energy consumption is not necessarily that 


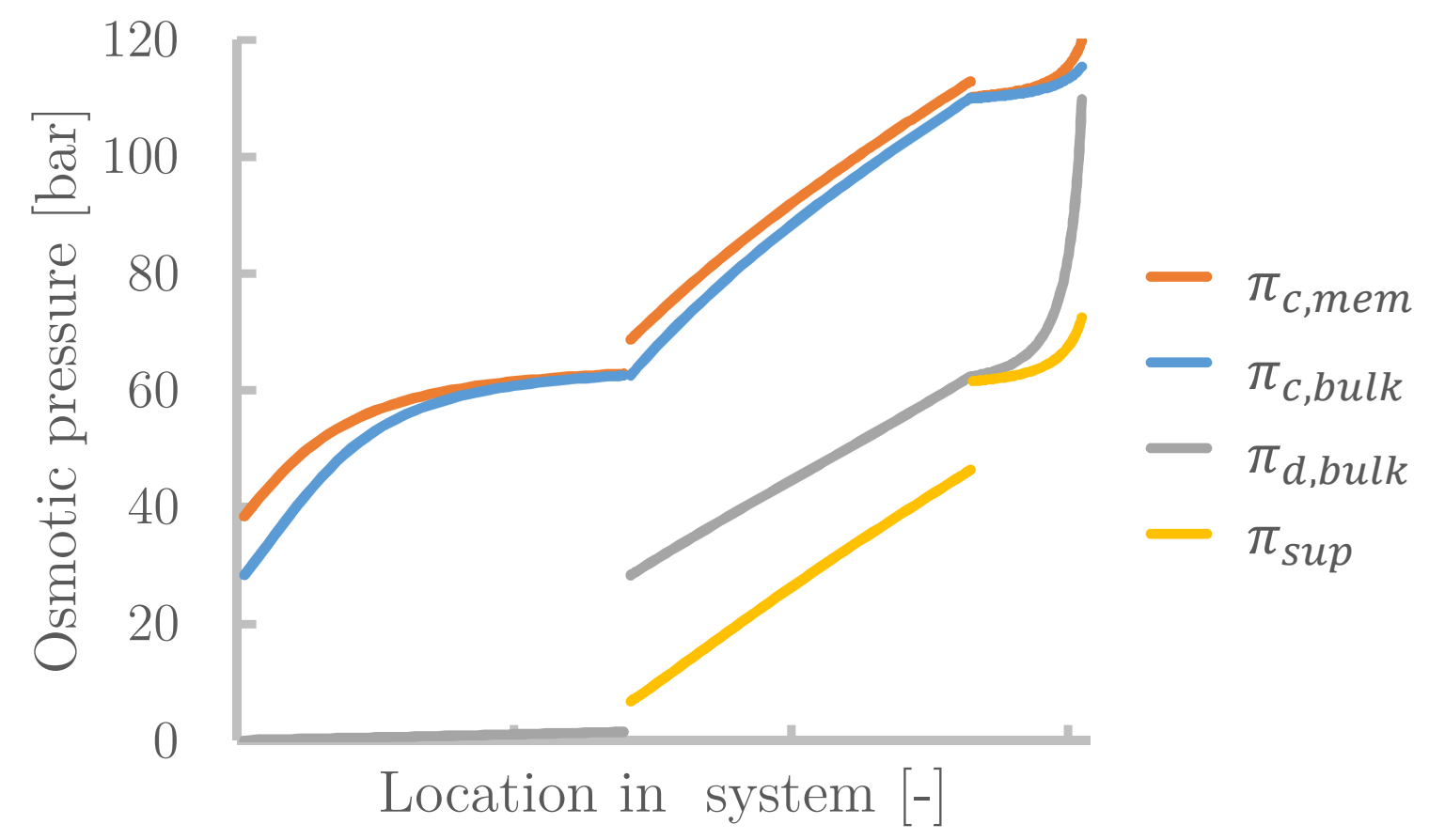

Figure 5-1: Osmotic pressures within an optimized 3-stage split-feed system, plotted over the nondimensionalized length of the system.

Table 5.1: System parameters within the optimized 3-stage split feed CFRO system.

\begin{tabular}{||c|c|c|c||}
\hline Stage & RO & CFRO 1 & CFRO 2 \\
\hline Applied pressure $[\mathrm{bar}]$ & 58 & 70 & 50 \\
\hline Membrane area $\left[\mathrm{m}^{2}\right]$ & 219 & 160 & 71 \\
\hline Flux $\left[\mathrm{L} / \mathrm{m}^{2}-\mathrm{h}\right]$ & 14.7 & 5.6 & 0.8 \\
\hline Inlet flow rate $[\mathrm{cm} / \mathrm{s}]$ & 15 & 8 & 4 \\
\hline
\end{tabular}

for which the system is equipartitioned. If the system were optimized when entropy generation within the membrane module was equipartitioned, as described in Section 3.2.1, we would expect the osmotic pressure difference across the membrane to be more or less constant over the length of the membrane in both CFRO stages, resulting in a balanced flux over the length. However, the system instead finds its minimum energy consumption when the osmotic pressure difference across the membrane is imbalanced. Clearly, unbalanced entropy generation plays a secondary role relative to other factors in this system for minimizing the whole system's entropy generation. Our previous exergy destruction analysis showed us that the majority of the inefficiency in the system was the result of entropy generation in the pumps and depressurization in the membrane. One possible reason that the system is optimized in the way shown is 
that it reduces the volume that must be pumped. By creating a much larger change in concentration in the first stage, the volume passed to the second CFRO stage is very small, which means that much less pump work is required to be done further along in the system. However, if the total change in concentration is split more evenly between the two CFRO stages, a larger volume is required to be pumped and flow through the membrane in both the first and second stages, which causes more entropy generation.

Another way to think about this is that if the RO system requires a fixed amount of additional feed solution in order to achieve the desired overall recovery ratio, and this additional feed comes from the first CFRO stage's diluate outlet, then if the first stage operates at a lower recovery ratio, more liquid needs to be circulating in the CFRO portion of the system in order to produce the required amount of additional $\mathrm{RO}$ feed. A lower recovery ratio in the first CFRO stage must be countered by a larger recovery ratio in the second CFRO stage, which results in more solution recirculating through the system in the loop shown in Figure 5-2. While a larger amount of fluid

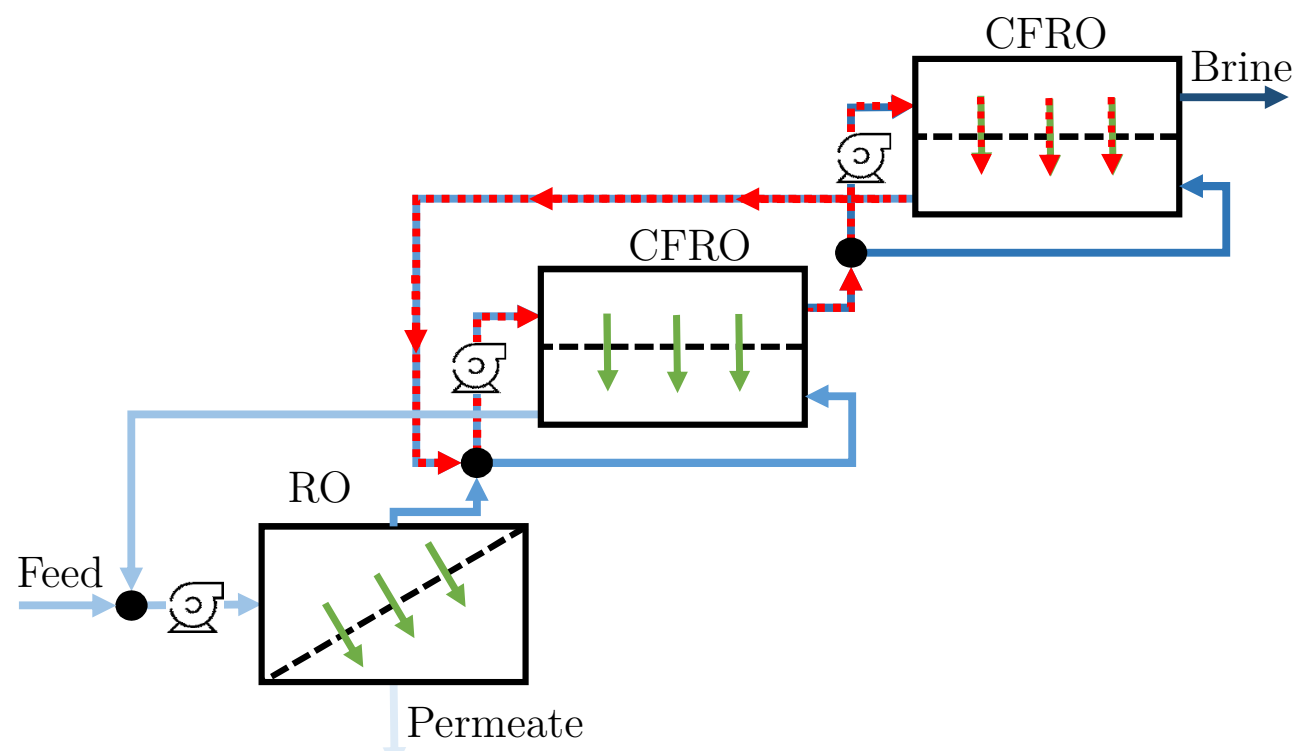

Figure 5-2: Diagram of a 3-stage split-feed CFRO system. The red dashed streams show a path of liquid recirculation. Increasing the flow rate in this loop can help to balance out the fluxes in the CFRO stages, but also increases pump work.

recirculating in this loop acts to balance the entropy generation in each individual 
stage spatially, it also increases the overall entropy generation in the system, as a result of pumping the recirculated liquid and the recurrent passage of water through the membranes.

We would like to expand this analysis to a much wider range of systems, including systems operating at different recovery ratios and number of stages. However, as more stages are added, the number of optimization variables grows as well, greatly increasing computation time to the point that a full optimization is time prohibitive. To reduce the number of degrees of freedom, we first seek to understand which variables make the largest difference when minimizing specific energy consumption. A sensitivity study shows that the specific energy consumption is most strongly affected by the membrane areas of each stage and the RO outlet concentration; other variables have a weaker influence. Modifying the membrane areas affects the flux in each stage, which can greatly affect the degree of concentration polarization seen by the system, and thus the pressure that is required in each stage. The RO outlet concentration determines the amount of work done by the RO section versus the CFRO section of the system. Increasing the RO outlet concentration will increase the flux and reduce the efficiency in the RO system, and decrease the amount of work that must be done in the CFRO system, as well as the volume of fluid in the CFRO system in general.

Using this information, we set up simulations with a reduced number of free variables. The goal here is not to determine how to best hybridize RO and CFRO systems in terms of distributing work between the two systems, but to characterize the performance of RO-CFRO hybrid systems using readily available RO technologies. To isolate the RO system while CFRO systems are changing, we fix the RO systems inlet flow rate at $10 \mathrm{~cm} / \mathrm{s}$, its membrane flux at $15 \mathrm{~L} / \mathrm{m}^{2}-\mathrm{h}$, and its outlet concentration at $70 \mathrm{~g} / \mathrm{kg}$, which also fixes the RO stage's recovery ratio at $50 \%$. With these constraints, we can isolate any benefits of operating in tandem with CFRO to the benefits generated by the CFRO portion of the system, not any benefit that comes with changing the operating parameters of the RO system itself. Additionally, with these constraints in place, for any desired outlet concentration or overall system recovery ratio, the amount of additional $\mathrm{RO}$ feed required to be produced by the CFRO 
stages as well as the flow rate of RO concentrate are fixed.

In order to compare systems of various sizes, we define a new variable called the CFRO specific flux. This variable is the volumetric flow rate of additional RO feed produced by the CFRO system divided by the total membrane area in all CFRO stages

$$
\text { Flux }_{\mathrm{CFRO}}=\frac{\dot{V}_{\text {feed }, R O}}{\sum_{1}^{N} A_{m e m, i}}
$$

where $N$ is the number of CFRO stages. This variable allows us to specify the relative size of the system, and reduces the number of degrees of freedom by one. To further reduce the number of unconstrained variables, we set the concentrate inlet flow rate in every CFRO stage to $10 \mathrm{~cm} / \mathrm{s}$. The sensitivity analysis shows that this variable does not have a large effect on the overall energy consumption in the system, and 10 $\mathrm{cm} / \mathrm{s}$ is on the order of flow rates common in RO systems.

The last variables that we fixed prior to optimizing the system are the intermediate concentrations between CFRO stages. The previous simulations have shown that performing the majority of the concentration in the first CFRO stages is advantageous, and the sensitivity analysis has shown us that the intermediate concentrations are not the most important of the degrees of freedom with respect to minimizing specific energy consumption. Thus, we choose to set the intermediate concentrations using a logarithmic scale, dividing the total change in concentration required in the CFRO stages so that the first stage always sees the largest change in concentration. Further inspection of the previous simulations also shows that the effects of ECP on the diluate side of the membrane are negligible compared to ICP within the operating range we examine here, and so this effect is neglected for the following simulations.

With these constraints imposed, the only remaining variables to be optimized are the size of the membrane areas for the CFRO stages. Since the overall area for the CFRO stages is set by the CFRO specific flux, the number of free variables is the number of CFRO stages minus one. Thus, for a three stage system with two CFRO stages, there is only one free variable, and for a six stage system with five CFRO stages, which is shown in Figure 5-3, there are four free variables. These variables are 


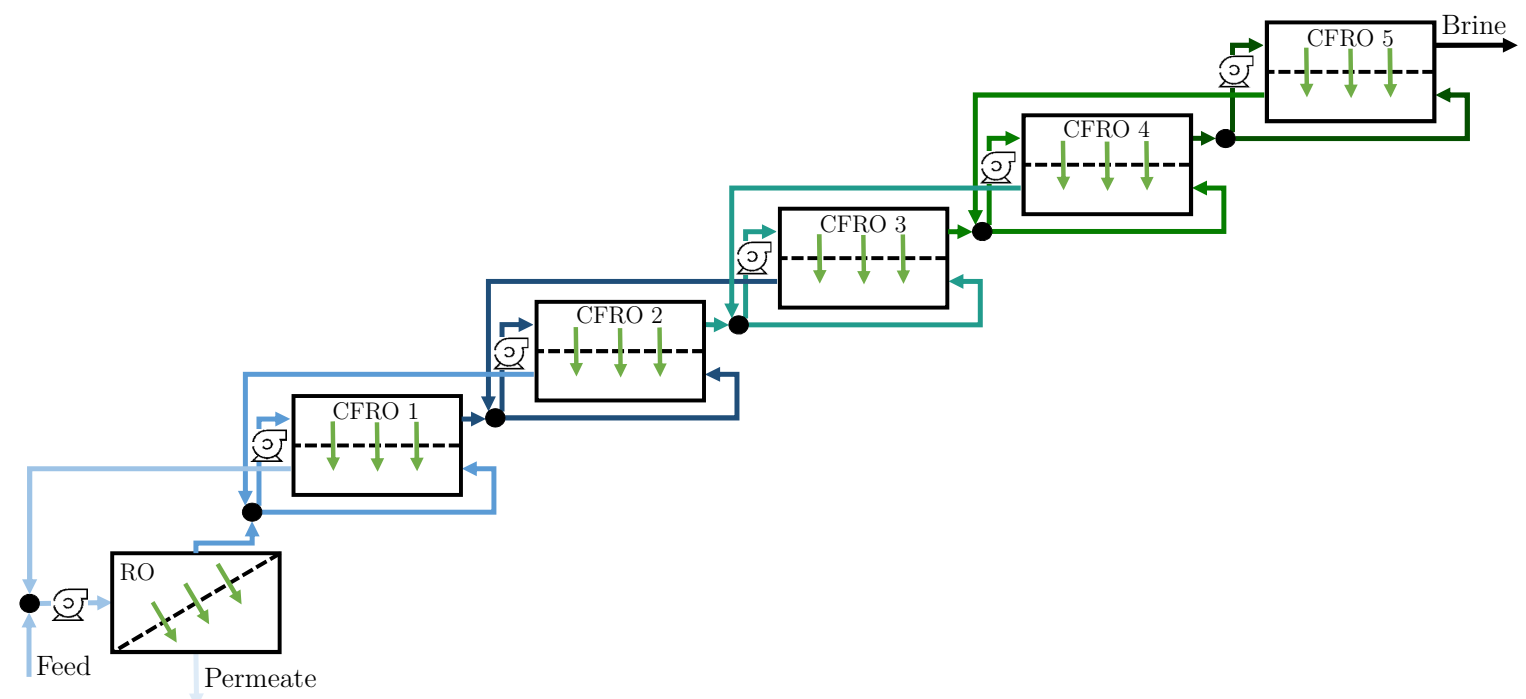

Figure 5-3: Diagram of a 6-stage split-feed CFRO system.

optimized using the methods described in Section 2.9 while minimizing the systems specific energy consumption. 


\section{Chapter 6}

\section{Results and Discussion}

\subsection{Multistaged system results}

The results of the simulations described in the previous section are shown in Figures 6-1 and 6-2 for varying CFRO specific fluxes and recovery ratios. Included in the figures for comparison are results for a single-stage reverse osmosis system operating at varying fluxes.

These results show the increased range of operation made possible by adding CFRO brine concentrators to existing RO systems. While normal RO systems are limited to a recovery ratio of around $60 \%$, CFRO brine concentration allows the system as a whole to recover over $80 \%$ of the feed. The six-stage configuration operating at a CFRO flux of $1 \mathrm{~L} / \mathrm{m}^{2}-\mathrm{h}$, with one RO stage and 5 CFRO stages, is able to concentrate the final brine solution to over $200 \mathrm{~g} / \mathrm{kg}$ while all hydraulic pressures in the system remain under 70 bar. A standard RO system would require a pressure above 180 bar to produce a brine of the same concentration. While high pressure RO, or conventional reverse osmosis operating at pressures above 70 bar, which abbreviated as HPRO, may be able to operate at reduced energy consumption compared to standard RO systems combined with $\mathrm{CFRO}$, CFRO systems may have additional benefits, such as operating at lower pressure differentials and fluxes, which may help to reduce the rate of fouling and scaling.

These results also confirm what we have shown in Section 3.2.1, that operating at 


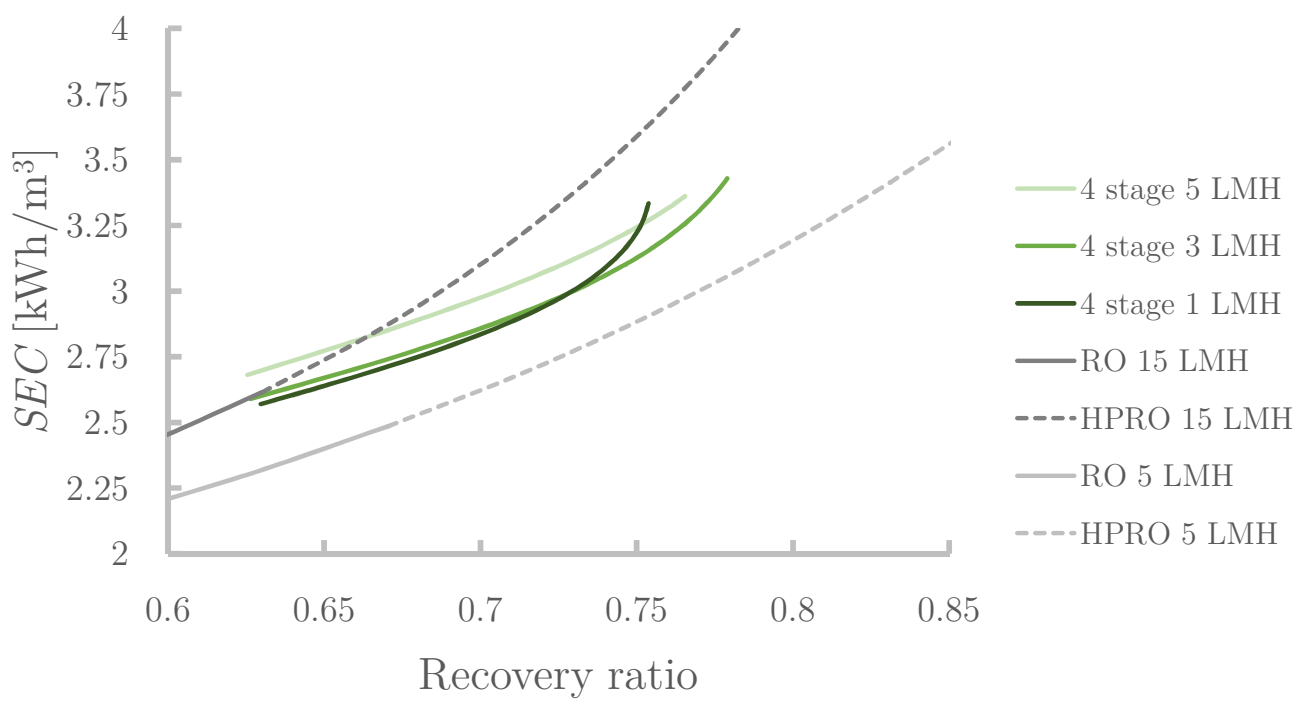

(a) 4-stage split feed CFRO

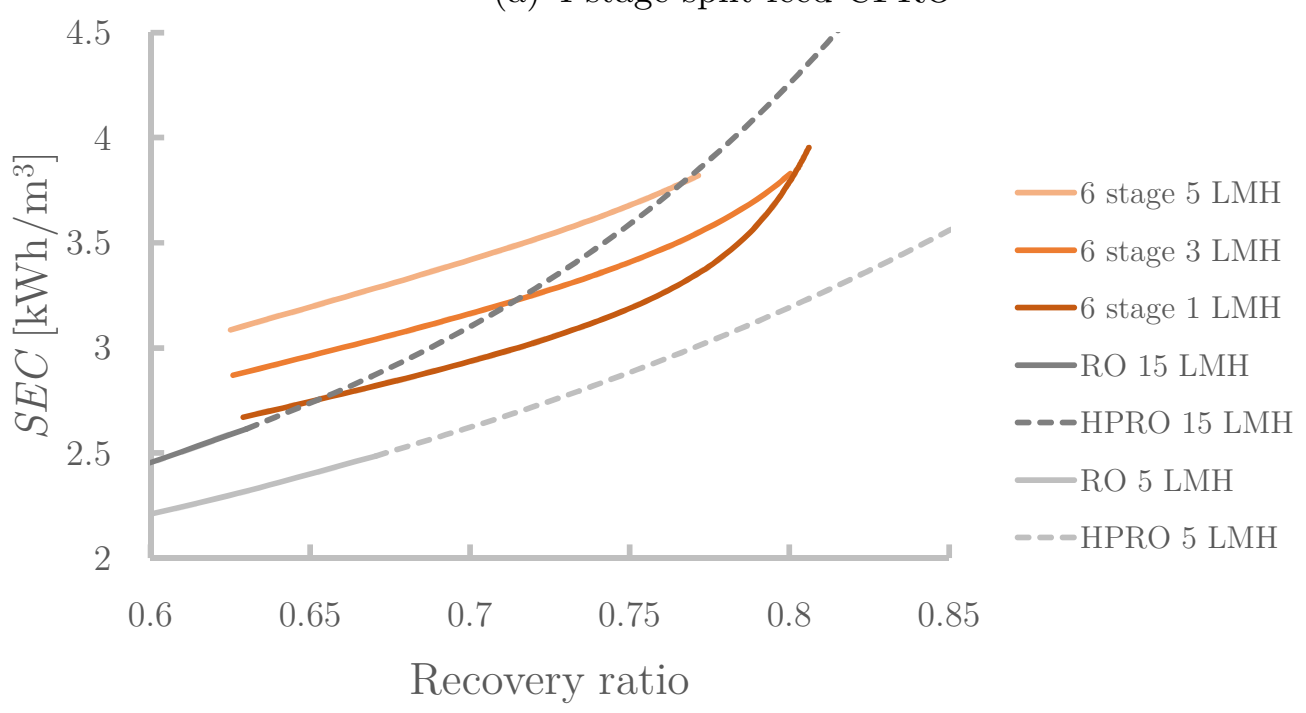

(b) 6-stage split feed CFRO

Figure 6-1: Specific energy consumption (SEC) for various recovery ratios and system sizes. High Pressure RO, labeled HPRO, shows to projected performance for conventional RO systems operating at pressures of greater than 70 bar. 


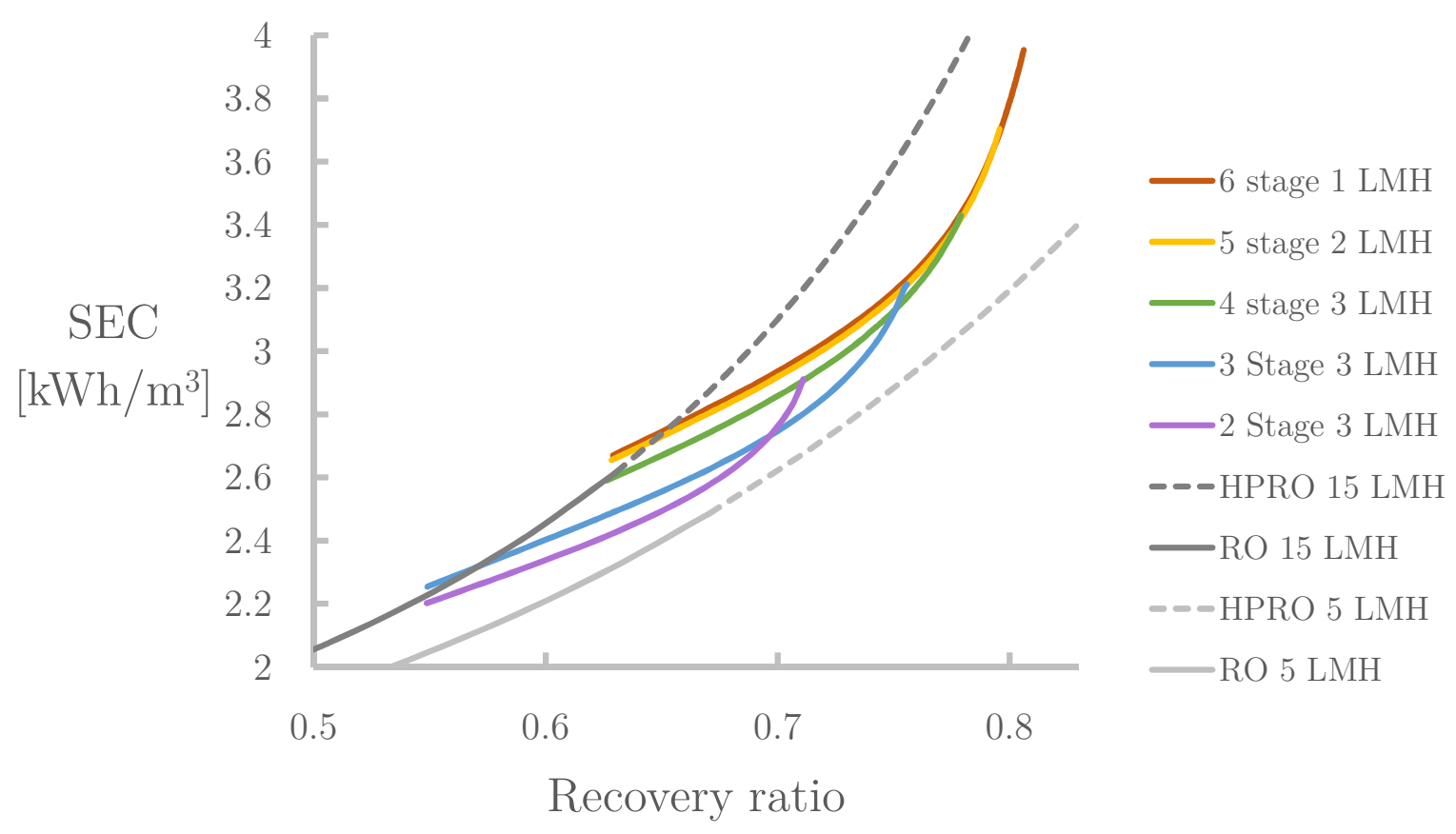

Figure 6-2: Performance of selected systems, selected to show the most energy efficient configuration for each recovery ratio. High Pressure RO, labeled HPRO, shows projected performance for conventional RO systems operating at pressures of greater than 70 bar.

very large system sizes is not always beneficial. At lower recovery ratios, systems that operate at a CFRO flux of $3 \mathrm{~L} / \mathrm{m}^{2}$-h consume less energy than systems operating at $1 \mathrm{~L} / \mathrm{m}^{2}$-h, because the smaller system with the higher flux experiences less entropy generation due to drag, while the effects of concentration polarization are still small at these operating conditions. At even higher fluxes and smaller system sizes, entropy generation due to drag becomes negligible compared to losses from concentration polarization. Therefore, for each given recovery ratio, there is an optimal system size that balances the losses due to these two effects.

Further, the results show that adding additional stages to the system does not always improve efficiency, and for the specific conditions used here, adding additional stages often hurts performance. While additional stages allow for improved balancing within each stage, each additional stage requires an extra pump and energy recovery device, each of which generate irreversibilities. Each additional stage also provides another opportunity for fluid to recirculate in a loop, which causes entropy generation each time the fluid recirculates without providing useful net output. While adding 
more stages does not always help to reduce energy consumption, it does help to increase the maximum recovery ratio that can be achieved by a system.

Using the setup described above, with the concentrations fixed at all points and specified feed conditions, one consequence is that the mass flow rates at all points in the system are also fixed as well. Thus, by varying the membrane area within each stage, optimization occurs by minimizing the entropy generation due to concentration polarization throughout the system, not by modifying any flow rates. Entropy generation is minimized by having increased fluxes in the first stages, where the salinity is low and losses due to concentration polarization are less significant. This allows the higher salinity final stages to operate at lower fluxes, which is beneficial because concentration polarization becomes a larger factor at high concentrations. This trend is illustrated in Table 6.1, where the flux in each stage of a 6-stage system is shown.

Table 6.1: Operating parameters for a 6-stage split-feed system concentrating up to $200 \mathrm{~g} / \mathrm{kg}$

\begin{tabular}{||c|c|c|c|c||}
\hline Stage & $P[\mathrm{bar}]$ & Flux $\left[\mathrm{L} / \mathrm{m}^{2}-\mathrm{h}\right]$ & Area $\left[\mathrm{m}^{2}\right]$ & $w_{c, \text { in }}[\mathrm{g} / \mathrm{kg}]$ \\
\hline \hline RO & 56 & 15 & 241 & 35 \\
\hline 1 & 61 & 2.50 & 637 & 70 \\
\hline 2 & 68 & 1.25 & 464 & 120 \\
\hline 3 & 52 & 0.64 & 462 & 150 \\
\hline 4 & 44 & 0.43 & 354 & 171 \\
\hline 5 & 33 & 0.14 & 557 & 187 \\
\hline
\end{tabular}

Additionally, we compare the expected performance of a 6-stage CFRO system that concentrates brine to $200 \mathrm{~g} / \mathrm{kg}$, corresponding to a recovery ratio of $82 \%$, to a 6-effect MVC system operating at the same conditions [48]. In Table 6.2, we see a large reduction in energy consumption and a large improvement in $2^{\text {nd }}$ law efficiency by performing brine concentration with a work-driven system as opposed to an evaporative system.

From this analysis, we can conclude that hybridizing CFRO with RO is useful when the solutions being processed are too concentrated for RO to treat. RO will likely always be more efficient than RO-CFRO hybrids at low recovery ratios for the same system size because of the losses caused by internal concentration polarization, 
Table 6.2: Comparison of a 6-stage split feed CFRO system and 6-effect MVC system operating at the same conditions, both concentrating solutions from $35 \mathrm{~g} / \mathrm{kg}$ up to $200 \mathrm{~g} / \mathrm{kg}$

\begin{tabular}{||c|c|c|c||}
\hline System & Stages/effects & SEC $\left[\mathrm{kWh} / \mathrm{m}^{3}\right]$ & $\eta_{I I}$ \\
\hline \hline Split feed CFRO & 6 & 3.9 & $49 \%$ \\
\hline MVC & 6 & 14.1 & $13 \%$ \\
\hline
\end{tabular}

which are not present in RO systems. However, when RO must operate near its maximum recovery ratio and losses due to ECP are large, it may be possible for CFRO to improve the system's efficiency. At concentrations which are too high for RO to treat, split feed CFRO is a viable option for brine concentration, and likely a significant improvement over existing evaporative brine concentration technologies.

Further improvements to the system can be made in the future by allowing intermediate concentrations at each intermediate point to be optimized, which would help to further reduce overall entropy generation by finding a balance between reducing the effects of concentration polarization and reducing the recirculating fluid. Improvements can also be made to the system by increasing component efficiency and developing improved membranes specifically for CFRO operation, optimizing flow velocities throughout the system, or by allowing the recovery ratio of the RO system to change.

\subsection{Varying $B$ in a 6-stage system}

As was discussed in Section 3.2.2, it cannot be determined by looking at a single stage's operation whether or not having a membrane with a higher or lower $B$ value is beneficial, because changing the $B$ value changes the system being analyzed. While operation at a high $B$ does not have a thermodynamic benefit, there still may be some real-world benefit to having a "leaky" membrane. Although efficiency is sacrificed, if the flux increases so significantly that the desired chemical separation can be accomplished using a plant with a smaller footprint, less membrane area, and reduced capital costs, a leaky membrane may be beneficial. Here, we take the 6-stage sys- 
tem described above, operating with a feed concentration of $35 \mathrm{~g} / \mathrm{kg}$ and an outlet concentration of $200 \mathrm{~g} / \mathrm{kg}$, and allow the salt permeability to vary. These results are shown in Figure 6-3.

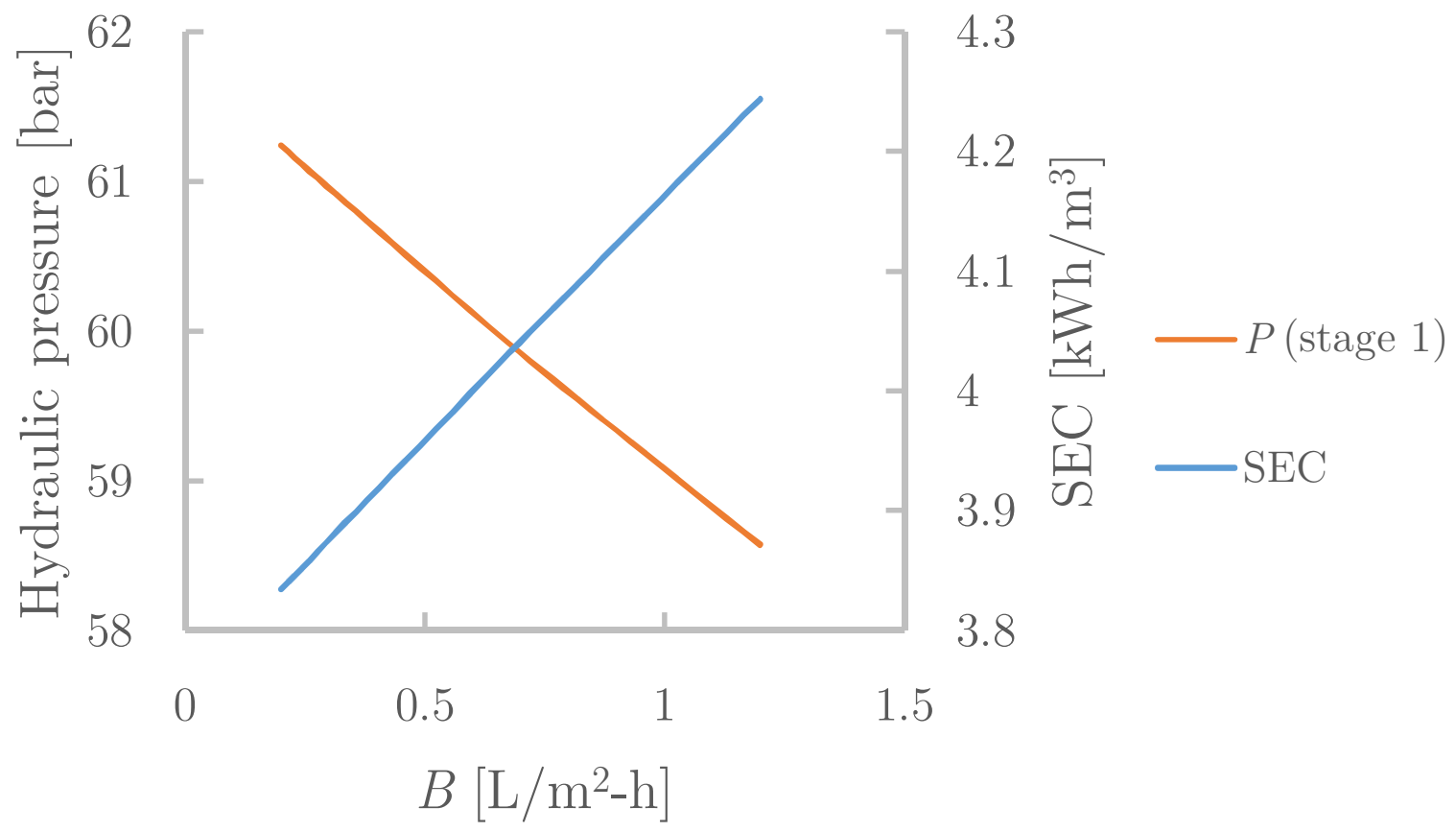

Figure 6-3: Change in specific energy consumption (SEC) and applied hydraulc pressure in the first CFRO stage as the membrane's salt permeability $(B)$ is varied.

Because in this case we have a system of fixed size, the reduced effects of ICP are apparent in the lower applied pressures within the system. As the salt permeability increases, the required energy consumption increases as before, while the applied pressures within the system are reduced. Again, this result is inconclusive, as what we have shown here is that a smaller system could be used to perform the operation if "leaky" membranes are implemented, but the operating costs will rise. Whether or not a leaky membrane is beneficial for any given case will depend on the ratio of the fixed costs of the system to the operating costs associated with purchasing additional power. 


\section{Chapter 7}

\section{Summary and Conclusions}

Brine concentration is becoming an increasingly important part of a complete water treatment system. Drivers such as tighter regulations, costs of disposing of waste, and environmental concerns are all encouraging alternative methods of waste disposal, including waste reduction by brine concentration. While effective methods exist, existing technologies are often energy intensive. Recent work has shown that significant energy reductions are possible when using membrane-based brine concentration as opposed to evaporative methods [22, 23].

By examining the operation of a single module under a variety of conditions, we have determined that membranes constructed specifically for CFRO should seek to have a high permeability, low structural parameter, and should tune salt permeability for maximum cost effectiveness. Purposefully developing "leaky" membranes may seem counterintuitive, but the reductions in ICP that result from allowing salt to pass through the membranes can significantly increase flux and allow for a system to operate with reduced membrane area, and thus reduced capital costs. It remains to be seen how designing membranes that allow salt to flow through more freely will affect fouling, scaling, membrane cleaning, and other operating characteristics of the system as a whole.

The split-feed configuration introduced in this paper has the significant benefit of not having limitations on maximum recovery ratio that are inherent to the configuration itself. Any limitations on recovery will come from other factors such as fouling 
and scaling, cost constraints, or inability of materials to handle high salinity conditions. The additional degrees of freedom in the system allow for flexible operation at a wide variety of conditions. The configuration shows the potential to operate very efficiently in a variety of regimes, and we have shown significant improvements in energy usage compared to thermal and evaporative brine concentration technologies.

Entropy generation in split-feed CFRO systems will be minimized when the change in concentration in each stage is weighted such that the initial stages, operating at lower concentrations, facilitate larger changes in concentration than the later stages, which operate at higher concentrations. Although this does not minimize the spatial variance in entropy generation within a system, it does reduce entropy generation by recirculating less liquid through entropy-generating processes and components.

Problems that still need to be investigated and addressed include operation of membrane-based brine concentration at very high concentrations, development of CFRO-specific membranes, and understanding the costs of CFRO systems compared to more established technologies. It is unclear to what extent fouling and scaling may be accelerated in CFRO systems at the relevant operating conditions. Recent work suggests that high pressure per se does not affect fouling [24], and CFRO experiments are needed in order to better understand fouling and scaling for these low pressure, high salinity systems. Finally, cost modeling for CFRO systems needs to be performed to better understand how competitive this technology can be with other brine concentration technologies. Due to the lower-flux requirements of CFRO to avoid losses from ICP, it is assumed that membrane areas will need to be large. Additionally, systems with many stages will require many additional components and complex control systems, driving up capital costs. These additional costs will have to be compared to the potential energy savings from reduced energy consumption to determine the conditions that make CFRO technology feasible. 


\section{Bibliography}

[1] José M. Estrada and Rao Bhamidimarri. A review of the issues and treatment options for wastewater from shale gas extraction by hydraulic fracturing. Fuel, 182:292-303, oct 2016 .

[2] Tiezheng Tong and Menachem Elimelech. The Global Rise of Zero Liquid Discharge for Wastewater Management: Drivers, Technologies, and Future Directions. Environmental Science and Technology, 50(13):6846-6855, jul 2016.

[3] Mark Heuer and Shan Yan. Marcellus Shale Fracking and Susquehanna River Stakeholder Attitudes: A Five-Year Update. Sustainability, 9(12):1713, sep 2017.

[4] Biplob Kumar Pramanik, Li Shu, and Veeriah Jegatheesan. A review of the management and treatment of brine solutions. Environ. Sci.: Water Res. Technol, 3,2017 .

[5] Benjamin K. Sovacool. Cornucopia or curse? Reviewing the costs and benefits of shale gas hydraulic fracturing (fracking). Renewable and Sustainable Energy Reviews, 37:249-264, sep 2014.

[6] Brian D. Lutz, Aurana N. Lewis, and Martin W. Doyle. Generation, transport, and disposal of wastewater associated with Marcellus Shale gas development. Water Resources Research, 49(2):647-656, feb 2013.

[7] Lauren A. Patterson and Kelly O. Maloney. Transport of hydraulic fracturing waste from Pennsylvania wells: A county-level analysis of road use and associated road repair costs. Journal of Environmental Management, 181:353-362, oct 2016.

[8] Paripurnanda Loganathan, Gayathri Naidu, and Saravanamuthu Vigneswaran. Mining valuable minerals from seawater: a critical review. Environ. Sci.: Water Res. Technol, 3(37), 2017.

[9] Richard G. Taylor, Bridget Scanlon, Petra Döll, Matt Rodell, Rens van Beek, Yoshihide Wada, Laurent Longuevergne, Marc Leblanc, James S. Famiglietti, Mike Edmunds, Leonard Konikow, Timothy R. Green, Jianyao Chen, Makoto Taniguchi, Marc F. P. Bierkens, Alan MacDonald, Ying Fan, Reed M. Maxwell, Yossi Yechieli, Jason J. Gurdak, Diana M. Allen, Mohammad Shamsudduha, Kevin Hiscock, Pat J.-F. Yeh, Ian Holman, and Holger Treidel. Ground water and climate change. Nature Climate Change, 3(4):322-329, apr 2013. 
[10] Steve Maxwell. Historical Water Price Trends. Technical report, American Water Works Assocation, 2010.

[11] C. J. Vörösmarty, P. B. McIntyre, M. O. Gessner, D. Dudgeon, A. Prusevich, P. Green, S. Glidden, S. E. Bunn, C. A. Sullivan, C. Reidy Liermann, and P. M. Davies. Global threats to human water security and river biodiversity. Nature, 467(7315):555-561, sep 2010.

[12] Karan H. Mistry, Ronan K. McGovern, Gregory P. Thiel, Edward K. Summers, Syed M. Zubair, and John H. Lienhard. Entropy Generation Analysis of Desalination Technologies. Entropy, 13(12):1829-1864, sep 2011.

[13] C Fritzmann, J Löwenberg, T Wintgens, and T Melin. State-of-the-art of reverse osmosis desalination. Desalination, 216:1-76, 2007.

[14] Quantum J. Wei, Ronan K. McGovern, and John H. Lienhard. Saving energy with an optimized two-stage reverse osmosis system. Environmental Science: Water Research \&5 Technology, 3(4):659-670, jun 2017.

[15] Richard L. Stover. Industrial and brackish water treatment with closed circuit reverse osmosis. Desalination and Water Treatment, 51(4-6):1124-1130, jan 2013.

[16] Jay R. Werber, Akshay Deshmukh, and Menachem Elimelech. Can batch or semi-batch processes save energy in reverse-osmosis desalination? Desalination, 402:109-122, jan 2017.

[17] David M. Warsinger, Emily W. Tow, Kishor G. Nayar, Laith A. Maswadeh, and John H. Lienhard. Energy efficiency of batch and semi-batch (CCRO) reverse osmosis desalination. Water Research, 106:272-282, dec 2016.

[18] David M. Warsinger, Emily W. Tow, Laith A. Maswadeh, Grace B. Connors, Jaichander Swaminathan, and John H. Lienhard. Inorganic fouling mitigation by salinity cycling in batch reverse osmosis. Water Research, feb 2018.

[19] H. Strathmann. Electrodialysis, a mature technology with a multitude of new applications. Desalination, 264(3):268-288, dec 2010.

[20] Shuaifei Zhao, Linda Zou, Chuyang Y. Tang, and Dennis Mulcahy. Recent developments in forward osmosis: Opportunities and challenges. Journal of Membrane Science, 396:1-21, apr 2012.

[21] Kevin W Lawson and Douglas R Lloyd. Membrane distillation. Journal of Membrane Science, 124:1-25, 1997.

[22] Timothy V. Bartholomew, Laura Mey, Jason T. Arena, Nicholas S. Siefert, and Meagan S. Mauter. Osmotically assisted reverse osmosis for high salinity brine treatment. Desalination, 421:3-11, nov 2017. 
[23] Xi Chen and Ngai Yin Yip. Unlocking High-Salinity Desalination with Cascading Osmotically Mediated Reverse Osmosis: Energy and Operating Pressure Analysis. Environmental Science $\&$ Technology, page acs.est.7b05774, feb 2018.

[24] Emily W. Tow and John H. Lienhard. Unpacking compaction: Effect of hydraulic pressure on alginate fouling. Journal of Membrane Science, 544:221-233, dec 2017.

[25] J.G. Wijmans and R.W. Baker. The solution-diffusion model: a review. Journal of Membrane Science, 107(1-2):1-21, nov 1995.

[26] G. Prakash Narayan, John H. Lienhard, and Syed M. Zubair. Entropy generation minimization of combined heat and mass transfer devices. International Journal of Thermal Sciences, 49(10):2057-2066, oct 2010.

[27] John H. Lienhard. Entropy Generation Minimization for Energy Efficient Desalination. In Proc. ASME 2018 International Mechanical Engineering Congress E Exhibition, Pittsburgh, 2018.

[28] Gregory P. Thiel, Emily W. Tow, Leonardo D. Banchik, Hyung Won Chung, and John H. Lienhard. Energy consumption in desalinating produced water from shale oil and gas extraction. Desalination, 366:94-112, jun 2015.

[29] Karan Mistry and John Lienhard. Generalized Least Energy of Separation for Desalination and Other Chemical Separation Processes. Entropy, 15(6):20462080, may 2013.

[30] Adrian Bejan. Advanced Engineering Thermodynamics. John Wiley \& Sons, Inc., Hoboken, NJ, USA, sep 2016.

[31] Kishor G. Nayar, Mostafa H. Sharqawy, Leonardo D. Banchik, and John H. Lienhard. Thermophysical properties of seawater: A review and new correlations that include pressure dependence. Desalination, 390:1-24, jul 2016.

[32] Mostafa H Sharqawy, John H Lienhard, and Syed M Zubair. Thermophysical properties of seawater: a review of existing correlations and data. Desalination and Water Treatment, 16:354-380, 2010.

[33] P. S. Z. Rogers and Kenneth S. Pitzer. Volumetric Properties of Aqueous Sodium Chloride Solutions. Journal of Physical and Chemical Reference Data, 11(1):1581, jan 1982.

[34] G. Schock and A. Miquel. Mass transfer and pressure loss in spiral wound modules. Desalination, 64:339-352, 1987.

[35] Robert W. Field and Jun Jie Wu. Mass transfer limitations in forward osmosis: Are some potential applications overhyped? Desalination, 318:118-124, jun 2013. 
[36] M F Gruber, C J Johnson, C Y Tang, M H Jensen, L Yde, and C Hélix-Nielsen. Computational fluid dynamics simulations of flow and concentration polarization in forward osmosis membrane systems. Journal of Membrane Science, 379:488495, 2011.

[37] C.P. Koutsou, S.G. Yiantsios, and A.J. Karabelas. A numerical and experimental study of mass transfer in spacer-filled channels: Effects of spacer geometrical characteristics and Schmidt number. Journal of Membrane Science, 326(1):234251, 2009.

[38] Anthony P. Straub, Ngai Yin Yip, and Menachem Elimelech. Raising the Bar: Increased Hydraulic Pressure Allows Unprecedented High Power Densities in Pressure-Retarded Osmosis. Environmental Science \& Technology Letters, 1(1):55-59, jan 2014.

[39] DOW. Water \& Process Solutions, FILMTEC Reverse Osmosis Membranes: Technical Manual. Dow Chemical Company, page 181, 2013.

[40] L Song and K Tay. Performance prediction of a long crossflow reverse osmosis membrane channel. Journal of Membrane Science, 281(1-2):163-169, sep 2006.

[41] P Charbonneau. Genetic algorithms in astronomy and astrophysics. The Astrophysical Journal Supplement Series, 101:309-334, 1995.

[42] A.I. Radu, J.S. Vrouwenvelder, M.C.M. van Loosdrecht, and C. Picioreanu. Effect of flow velocity, substrate concentration and hydraulic cleaning on biofouling of reverse osmosis feed channels. Chemical Engineering Journal, 188:30-39, apr 2012.

[43] Tzahi Y Cath, Amy E Childress, and Menachem Elimelech. Forward osmosis: Principles, applications, and recent developments. Journal of Membrane Science, 281:70-87, 2006.

[44] Daniel Tondeur and Eric Kvaalen. Equipartition of Entropy Production. An Optimality Criterion for Transfer and Separation Processes. Industrial and Engineering Chemistry Research, 26(1):50-56, 1987.

[45] Gregory P. Thiel, Ronan K. McGovern, Syed M. Zubair, and John H. Lienhard. Thermodynamic equipartition for increased second law efficiency. Applied Energy, 118:292-299, apr 2014.

[46] Karim M. Chehayeb, Kishor G. Nayar, and John H. Lienhard. On the merits of using multi-stage and counterflow electrodialysis for reduced energy consumption. Desalination, 439:1-16, aug 2018.

[47] Ronan K. McGovern and John H. Lienhard. On the asymptotic flux of ultrapermeable seawater reverse osmosis membranes due to concentration polarisation. Journal of Membrane Science, 520:560-565, dec 2016. 
[48] Jaichander Swaminathan, Kishor G. Nayar, and John H. Lienhard. Mechanical vapor compression - Membrane distillation hybrids for reduced specific energy consumption. Desalination and Water Treatment, 57(55):26507-26517, nov 2016. 